The University of Southern Mississippi

The Aquila Digital Community

Master's Theses

Spring 5-2010

\title{
A Parameterization Study of Short Read Assembly Using the Velvet Assembler
}

Alex Christopher Elliot

University of Southern Mississippi

Follow this and additional works at: https://aquila.usm.edu/masters_theses

\section{Recommended Citation}

Elliot, Alex Christopher, "A Parameterization Study of Short Read Assembly Using the Velvet Assembler" (2010). Master's Theses. 474.

https://aquila.usm.edu/masters_theses/474

This Masters Thesis is brought to you for free and open access by The Aquila Digital Community. It has been accepted for inclusion in Master's Theses by an authorized administrator of The Aquila Digital Community. For more information, please contact Joshua.Cromwell@usm.edu. 


\section{A PARAMETERIZATION STUDY OF SHORT READ ASSEMBLY}

\section{USING THE VELVET ASSEMBLER}

by

\section{Alex Christopher Elliot}

\section{A Thesis}

Submitted to the Graduate School

of The University of Southern Mississippi

in Partial Fulfillment of the Requirements

for the Degree of Master of Science

\section{Approved:}

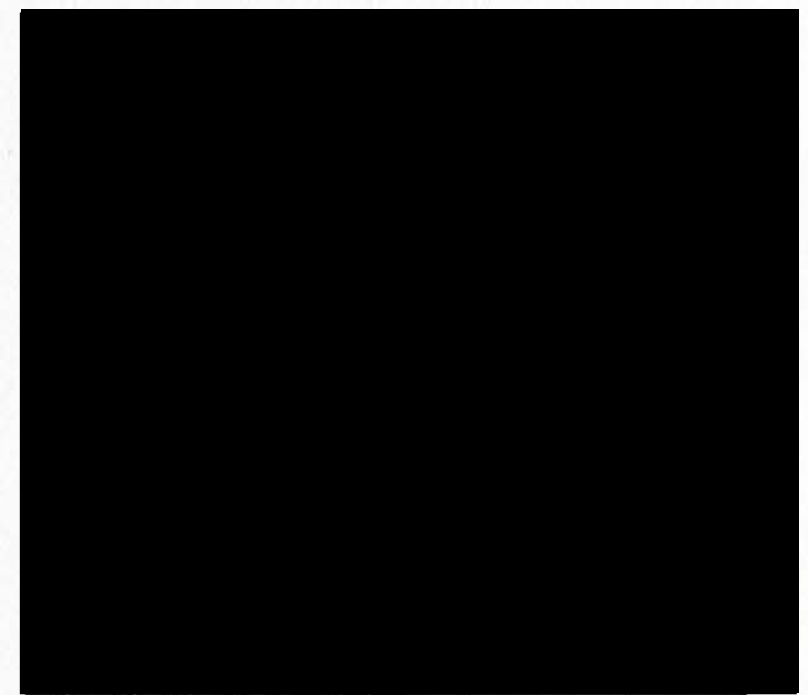

Dean of the Gradtuate School 


\begin{abstract}
A PARAMETERIZATION STUDY OF SHORT READ ASSEMBLY

USING THE VELVET ASSEMBLER

by Alex Christopher Elliot
\end{abstract}

May 2010

In this study, we examine approaches to the problem of assembling large, contiguous sections of genetic code from short reads generated from laboratory techniques. We explore the Eulerian Path approach in detail, utilizing a de Bruijn Graph, and demonstrate current software technologies and algorithms using a sample genome. We investigate the input parameters of Velvet and discuss choice implications in the context of the $\mathrm{E}$. coli putA/b1014 gene. 


\section{TABLE OF CONTENTS}

ABSTRACT ii

LIST OF ILLUSTRATIONS iv

LIST OF TABLES vii

CHAPTER

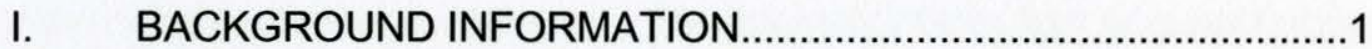

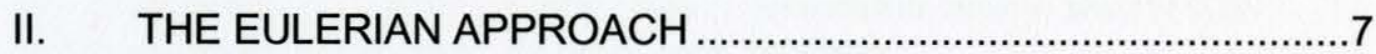

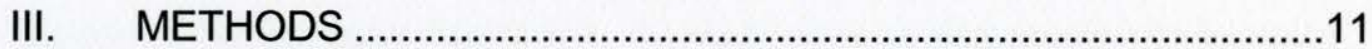

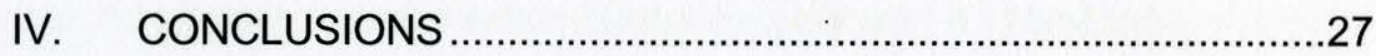

Future Work

APPENDIXES .29

REFERENCES .68 


\section{LIST OF ILLUSTRATIONS}

Figure

1. Sanger method overview. The Sanger method uses ddNTPs to terminate cloned genetic source material at each base location. The resultant strands are then separated by length via Gel Electrophoresis or Fluorescent Absorption and recorded. The Sanger method usually results in few, long $(\sim 1 \mathrm{kbp})$ reads (Applied Biosystems).

2. Pyrosequencing overview. Pyrosequencing introduces bases to source material iteratively. If a base binds to the source material, a measurable unit of light (photon) is released. The intensity of this light is quantifiable and relates to number of sequential bases encountered.

Pyrosequencing quickly ( $25 \mathrm{Mbp} / 4 \mathrm{hr}$ ) generates many; short (100$400 \mathrm{bp}$ ) reads (Marguiles, Egholm and Altman).

3. Steps in the Eulerian Approach. 1. Hash source sequence into k-mers of length k. 2. Group k-mers with overlap length of k-1 into nodes. 3. Nodes contain complimentary/mirror sequences. 4. Directed edges/arcs are formed to connect adjacent nodes where k-1 overlap is found. 5. Graph is simplified by condensing adjacent, single pathway nodes. "Tips" and "Bulges" are removed. 6. Error Correction. 7. Contig output sequence is path through series of connected nodes.

4. Assembly Programmatic Flow. Metasim generated simulated reads from source gene. These simulated read sets were hashed using velveth with varying k-mer lengths then assembled into de Bruijn graphs using velvetg.

5. Gene NP_415534 Physical Map. Gene NP_415534, putA was selected from the circular E. Coli chromosome to illustrate the assembly algorithms. This figure shows its physical location with reference to neighboring genes. (Kyoto University Bioinformatics Center)

6. Assembly Parameters (kmer/cvCut) vs. Indicators (Igth/N50). This scatter plot illustrates the effect of kmer length and coverage cutoff on N50 and assembly length. N50 refers to the length of the shortest contig in an assembly such that the sum of contigs of equal length or longer is at least $50 \%$ of the total length of all contigs. Here we see a logarithmic distribution where higher cvCut values generate larger contigs 
7. Assembly Parameters (kmer/cvCut) vs. Assembly Indicators (Ctg Count/N50). This plot shows the influence of kmer length and cvCut on the number of contigs produced with greater than $2^{*} k$ length. Again, we see a somewhat logarithmic function with higher cvCut and higher kmers producer longer and fewer isolated contigs.

8. Assembly Parameters (k-mer/cvCut) vs. Percent Read Usage. This figure compares k-mer and cvCut to the percent read usage. With sufficiently high $\mathrm{k}$, read utilization increases with coverage cutoff, due to the relaxation of selectivity of node removal.

9. Kernel density plot of log contig size distribution by controlling for overall contig count. It is interesting to note the bimodal nature of this data. Future research into the cause of this may uncover underlying information about the source data or function of the assembler. The highest kmer and cvCut runs appear to be more normal in distribution. 19

10. BLAST Map for Simulated 454 Reads $(k=31$, coverage cutoff $=12$, expected coverage $=24$ ). This figure, from BLAST, maps the 16 resultant contigs of the 454 simulated reads at k-mer length 31 , expected coverage 24 , and coverage cutoff 12 . The level of similarity to the reference gene is shown by the color, with red being the best quality. Contigs appear to high quality and well distributed about the gene.......20

11. BLAST Contig Scoring for Simulated 454 Reads $(k=21$, coverage cutoff $=2$, expected coverage $=4$ ). This assembly resulted in 4253 nodes and $\mathrm{n} 50$ of 16 . BLAST, maps the resultant contigs above a scoring threshold. The level of similarity to the reference gene is shown by the color, with red being the best quality. Contigs appear to be very small with medium to poor quality, yet well distributed about the gene.

12. BLAST Contig Scoring for Simulated "Exact" Reads $(k=21$, coverage cutoff $=2$, expected coverage $=4$ ). This assembly maps the single resultant contig of the simulated "exact" reads at k-mer length 21 , expected coverage 4 , and coverage cutoff 2 . This assembly resulted in a complete gene sequence regardless of input parameters, indicating that the importance of coverage cutoff and expected coverage lies mainly in error handling and correction. When the input was of complete consensus with the reference sequence, the output remained error free. The assembled contig achieved $100 \%$ reference gene coverage at $100 \%$ identity with a length of 3963 bases....................................................23

13. Hawkeye overview display. Simulated 454 simulated reads at k-mer length 31 , expected coverage 24 , and coverage cutoff 12 . Various statistics are shown, including a graph of the contig length distribution.24 
14. Hawkeye Contig Alignment Display. 454 simulated reads at k-mer length 31 , expected coverage 24 , and coverage cutoff 12 showing consensus alignments of the reads used to create one of the 16 generated contigs. This figure also shows the introduced errors in the simulated reads.

15. Hawkeye Contig Detail Display. 454 simulated reads at k-mer length 31, expected coverage 24, and coverage cutoff 12 showing information about each of the 16 generated contigs including length and number of reads used per contig. 


\section{LIST OF TABLES}

Table

1. Assembly Parameter Permutations.......................................14

2. BLAST Contig Scoring for Simulated 454 Reads $(k=31$, coverage cutoff $=12$, expected coverage $=24$ ) 21

3. BLAST Contig Scoring for Simulated 454 Reads $(k=21$, coverage cutoff $=2$, expected coverage $=4$ ) .22 


\section{CHAPTER I}

\section{BACKGROUND INFORMATION}

In order to fully understand the challenges and scope of read assembly, we must first discuss the biological and chemical background to the problem. All known living organisms and some viruses encode genetic information in the form of Deoxyribonucleic Acid (DNA) (Darnell, Lodish and Baltimore 66-74). DNA is a double helix shaped polymer consisting of two long, spiraling chains of alternating sugars and phosphate groups bonded to a series of nucleotides. These nucleotides, or bases, can be one of either adenine $(A)$ thymine $(T)$ guanine (G) or cytosine (C). Each nucleotide forms a bond with exactly one other base, known as its compliment, and the order of these bases houses the genetic information.

Through the processes of transcription and translation, an organism uses its genetic blueprint to first create ribonucleic acid (RNA). RNA is similar to DNA except that it contains uracil $(U)$ in the place of thymine $(T)$. Once a segment of DNA is translated to RNA, ordered amino acid chains are generated based on the RNA's nucleotide sequence. These amino acids are then assembled into proteins, which can range in size from tens to tens of thousands of amino acids each.

Each amino acid is encoded by three bases, and there are twenty possible amino acids in use by known organisms (Darnell, Lodish and Baltimore 88). Some areas of DNA also contain information to control gene regulation and expression as well as sections of "filler" data known as introns. With four 
possible bases, there are $4^{3}$, or 64 base combinations. Some amino acids have multiple coding triplets, and the information can be encoded in the forward or reverse direction. Furthermore, varying the start base can shift the read frame and thus the resulting codons, amino acids and proteins.

Eukaryotic cells such as those found in mammals and plants, encapsulate their DNA within a cell nucleus, often in chromosomes or tightly wound structures of DNA. Prokaryotic cells such as bacteria and archea without a true, membrane bound nucleus, store their genetic material in cytosol or intracellular fluid. The genetic material found in prokaryotes is normally arranged into smaller circular chromosomes. The collection of all genetic material contained in an organism is known as that organism's genome.

Since the discovery of the DNA double helix in 1953 (Watson and Crick, A Structure for Deoxyribose Nucleic Acid), science has sought to fully understand the information contained within it (Watson and Crick, Genetical Implications of the structure of Deoxyribonucleic Acid). In a macro view, understanding an organism's genome can help reveal its phylogeny and origins, while the micro view can uncover information about disease susceptibility and cure. Small sections of an individual organism's genetic fingerprint that indicate the presence or absence of a particular trait are called genetic biomarkers. These biomarkers can be used to, for example, determine relation between organisms, gauge exposure to a particular genetic toxicant, predict inherited disease, or determine an optimal treatment approach.

In order to understand genetic information, one must find a way to read 
the information contained within DNA or RNA. Genetic sequencing techniques were first developed in the early 1970's (Gilbert and Maxam). These complex methods, including the wandering-spot technique were very labor intensive.

Fredrick Sanger (Sanger, Nicklen and Coulson) and Gilbert (Maxam and Gilbert) independently published research in 1977 that greatly simplified the sequencing process. Both were awarded the Nobel Prize in 1980 for their work.

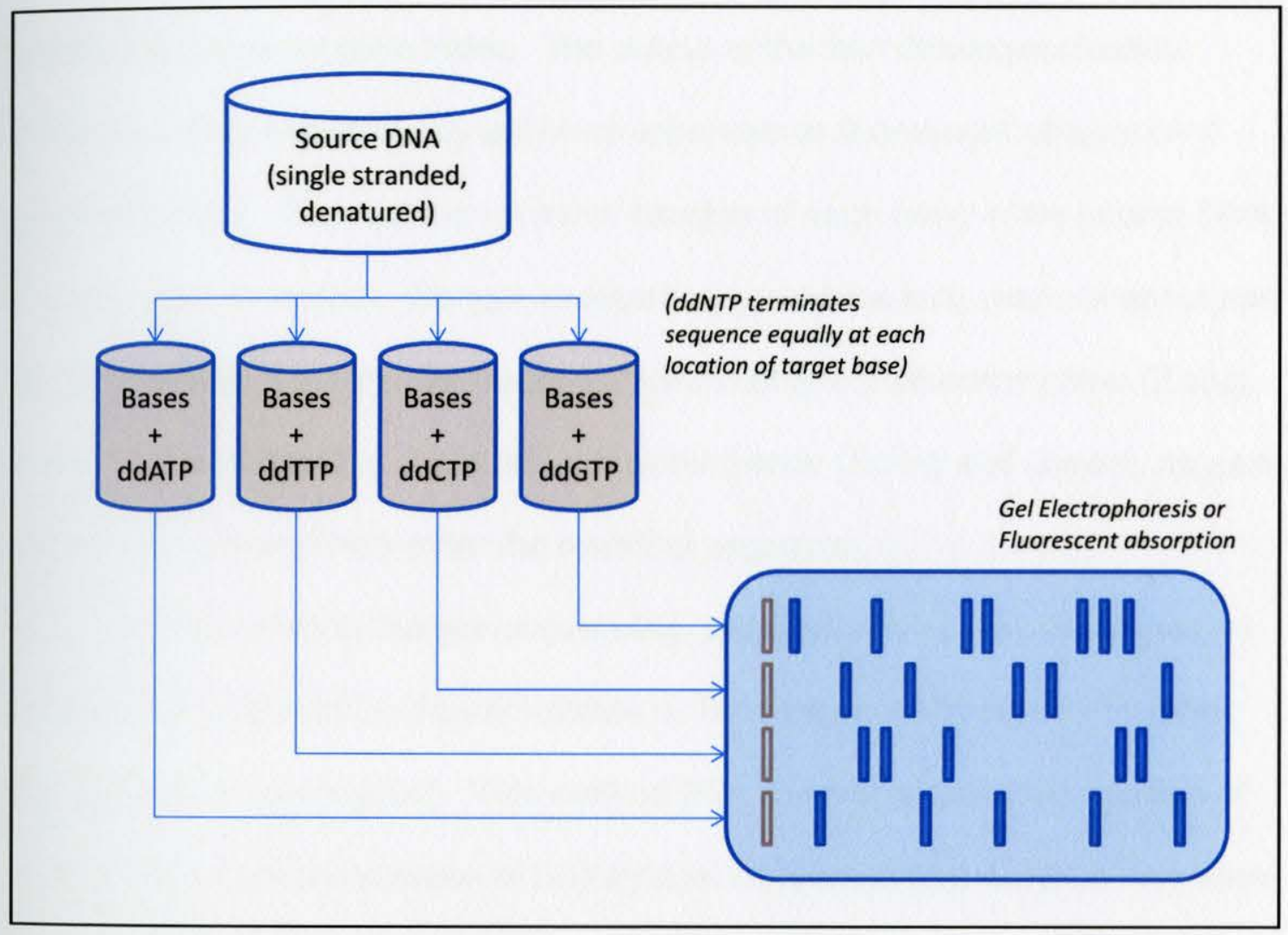

Figure 1: Sanger method overview. The Sanger method uses ddNTPs to terminate cloned genetic source material at each base location. The resultant strands are then separated by length via Gel Electrophoresis or Fluorescent Absorption and recorded. The Sanger method usually results in few, long ( $1 \mathrm{kbp}$ ) reads (Applied Biosystems). 
The Sanger method (Fig. 1) is a chain terminating technique that uses of dideoxynucleotide triphosphates (ddNTPs) to selectively terminate long strands of genetic material (Tamarin 334-6). In this method, single stranded, denatured DNA source material is cloned and separated into four separate solutions containing one of ddATP, ddTTP, ddCTP, or ddGTP each. The dideoxynucleotides terminate the multiple copies of the DNA strand at each location of the target base, resulting in strands that begin at the origin and have length of the base location index. The output of the four dideoxynucleotide solutions is then separated by gel electrophoresis or fluorescent absorption if dyes were used. The result is an index location of each base in the source DNA to a one-base resolution. Sanger sequencing generates long reads of about one thousand bases, but requires weeks to months of costly laboratory time (Ewing, et al.). This technique is susceptible to cloning error (Ewing and Green), as parts of the cloning vector may enter the resulting sequence.

An alternative to Sanger sequencing, pyrosequencing was developed by Nyrén and Ronaghi at the Royal Institute of Technology in Stockholm in 1998 (Ronaghi, Uhlén and Nyrén). This method (Fig. 2) involves iterative addition of bases in an enzymatic solution of Sulfurylase, Luciferase and Apryase. As each base bonds to the source material, a measurable amount of light is released per base. Repeat bases yield proportionately more light. After each base is introduced and bound, an enzyme is added to remove all unused bases before the next base is added. 

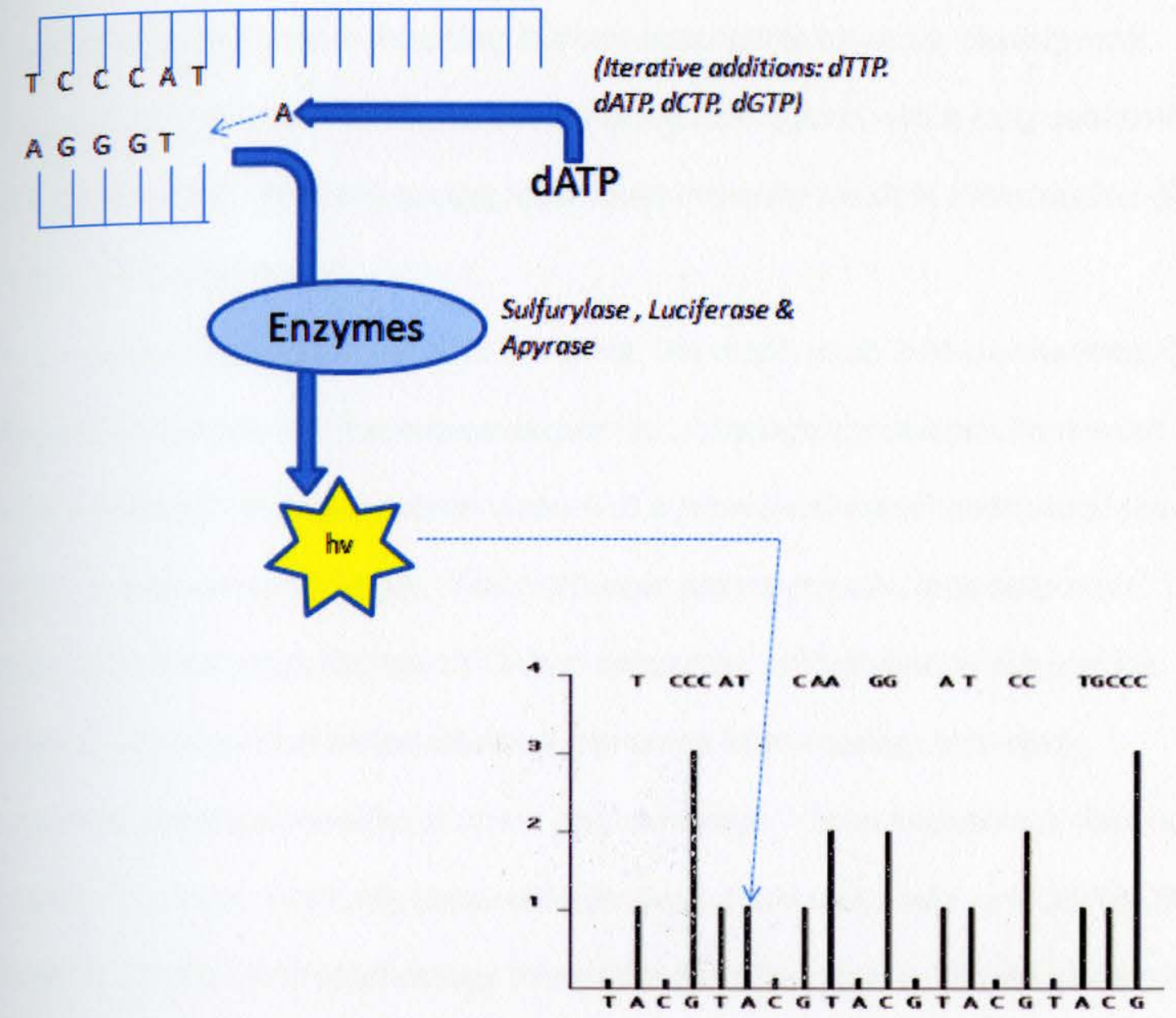

Figure 2: Pyrosequencing overview. Pyrosequencing introduces bases to source material iteratively. If a base binds to the source material, a measurable unit of light (photon) is released. The intensity of this light is quantifiable and relates to number of sequential bases encountered. Pyrosequencing quickly ( 25Mbp/4hr) generates many; short (100-400bp) reads (Marguiles, Egholm and Altman).

Pyrosequencing results in short length reads with an upper limit of approximately 500 bases; however commercial implementations are constantly increasing the maximum read length. Pyrosequencing is also less expensive to perform than traditional techniques, with companies such as 454 Life Sciences producing all-in-one units (Roche Diagnostics Co.). This technique can produce 
approximately $25 \mathrm{Mbp} / 4 \mathrm{hr}$ (Marguiles, Egholm and Altman). As this technique does not require traditional cloning, it is not susceptible to vector cloning error. It is, however, potentially less accurate in homopolar regions with a long series of repeating bases. Pyrosequencing techniques normally result in many copies of overlapping short reads.

After the laboratory work is complete, the reads must then be assembled into a representation of the source sequence. Although various solutions exist for this problem, all require some amount of a priori assumption and reliance on yet to be fully verified metrics. The challenge, algorithmically, is to determine how each of the reads fits into the larger sequence. Information to support the selection amongst candidate solutions can come from existing, reference genomes, statistical models, or sheer read coverage. Once sequenced, data can be added to large, publically accessible genome databases such as NCBI (NCBI [National Center for Biotechnology Information]) or GenomeNet (Kyoto University Bioinformatics Center).

The NCBI Basic Local Alignment Search Tool (BLAST) can be used to find regions of local similarity between sequences. The program (Altschul, Madden and Schaffer) compares nucleotide or protein sequences to sequence databases and calculates the statistical significance of matches. BLAST can be used to infer functional and evolutionary relationships between sequences as well as help identify members of gene families. 


\section{CHAPTER II}

\section{THE EULERIAN APPROACH}

In this section, we describe the application of the Eulerian path to short read assembly and its differences as compared to earlier methods. We will discuss one available implementation - Velvet, and provide insight into its algorithm.

Older approaches to the problem of read assembly were designed around the assembly of few, long reads. Many available programs utilized the "overlaplayout-consensus" paradigm which tests each possible read pair combination to determine the best matches. Each read is represented as a node, and each detected overlap is drawn as an arc between the overlapping nodes. Once matches are scored, the assembly is generated based on overlap scoring. Unfortunately, determining the layout leads to the NP-complete Hamiltonian Path Problem (Cormen, Leiserson and Rivest). The difficulty of the Hamiltonian Path Problem is exacerbated when attempting to operate on an increased number of reads.

Pevzner proposed an alternative solution to the read assembly problem for sequencing by hybridization (P. A. Pevzner). By making use of the de Bruijn Graph, he reduced read assembly to a solvable Eulerian Path Problem. Further work by Idury and Waterman (Idury and Waterman) applied the Eulerian path to short fragment assembly by treating short fragment assembly as a Sequencing by Hybridization problem. Pevzner, Tang and Waterman refined their Eulerian graph techniques in 2001 to include methods of error correction and repeat 
handling in data (Pevzner, Tang and Waterman).

An n-dimensional de Bruin graph contains vertices representing all sequence permutations of length $\mathrm{n}$ over a given alphabet with repeats (de Bruijn). For gene sequencing, our alphabet consists of a relatively few symbols A,T/U, G, C. Nodes are adjacent whenever they represent a one character shift left with any of the alphabet symbols shifted into the last location. Such a graph covers all possibilities. In read assembly algorithms, the de Bruin graph is built from the bottom up, adding vertices only when that permutation is found within the genetic information itself. This restricts the size of the graph, and also allows it to represent all possible overlap combinations for the underlying data.

Zerbino and Birney released a set of algorithms called "Velvet" (Zerbino and Birney) to manipulate de Bruijn graphs for genomic sequence assembly. In their implementation of the graph (Fig. 3), a $k$-mer is defined as a substring of length $k$, extracted from a read. Each node contains a series of overlapping kmers, with each overlap having length $k-1$ bases. Each node is attached to another, "mirror" node which contains the reverse series of $k$-mers. These mirror nodes take into account the complementary nature of genetic material. Nodes whose last $k$-mer overlaps with the first $k$-mer of another node are connected by a directed arc. The assembled contiguous sequence or "contig" is represented by a traversal from the first $k$-mer of the first node through connected arcs to each other node. 


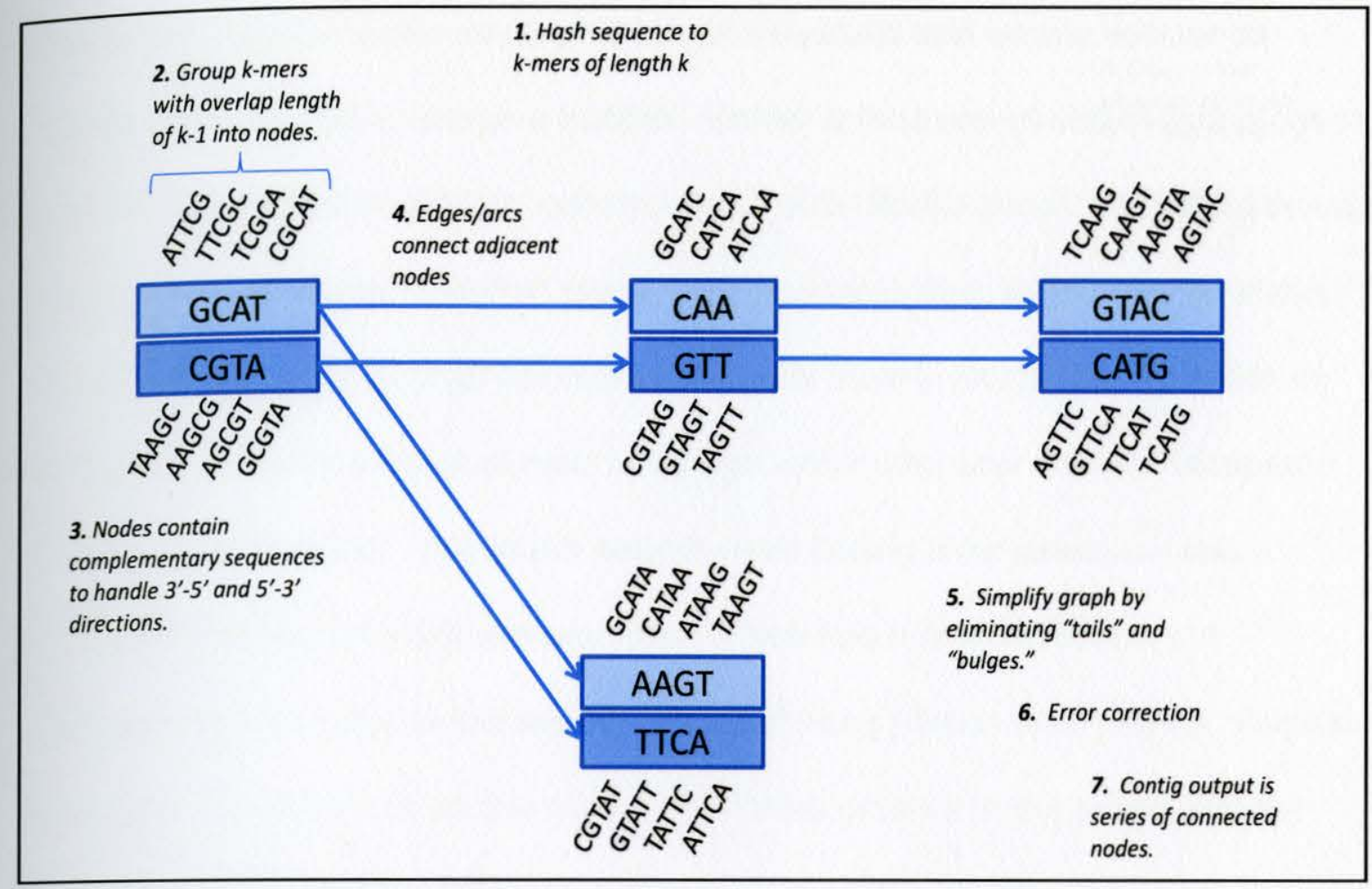

Figure 3: Steps in the Eulerian Approach. 1. Hash source sequence into k-mers of length k. 2. Group k-mers with overlap length of k-1 into nodes. 3. Nodes contain complimentary/mirror sequences. 4. Directed edges/arcs are formed to connect adjacent nodes where $\mathrm{k}-1$ overlap is found. 5. Graph is simplified by condensing adjacent, single pathway nodes. "Tips" and "Bulges" are removed. 6. Error Correction. 7. Contig output sequence is path through series of connected nodes.

Once the input reads have been hashed into $k$-mers and assembled into nodes and arcs, the resulting graph must be simplified and cleared of errors.

Velvet simplifies the graph by combining adjacent nodes with only one incoming and outgoing arc. This reduces the node count to only nodes with multiple arcs. Error correction is performed by eliminating "tips" and "bulges." A "tip" is defined as a chain of nodes connected at only one end, and Velvet removes tips that do not meet minimum length and coverage requirements. A "bulge" is a redundant path that starts and ends at the same nodes as other paths with similar 
sequences. Velvet again employs a length threshold and simple sequence identity to condense or merge a bubble. Velvet is thus composed of four stages: hashing the reads into $k$-mers, constructing the de Bruijn graph, correcting errors, and resolving repeats. The first stage, graph construction, is memory intensive. The time complexity of error correction depends mainly on number of nodes in the graph, which is a result of read coverage, error rate, and number of repeats in the source material. The graph search used during error detection and correction employs the Dijkstra algorithm which has a time complexity of $\mathrm{O}(\mathrm{N} \log \mathrm{N})$ when implemented with a Fibonacci heap (Gross and Yellen). Repeat resolution also depends on the number of nodes present in the graph and the average length of those nodes. 


\section{CHAPTER III}

\section{METHODS}

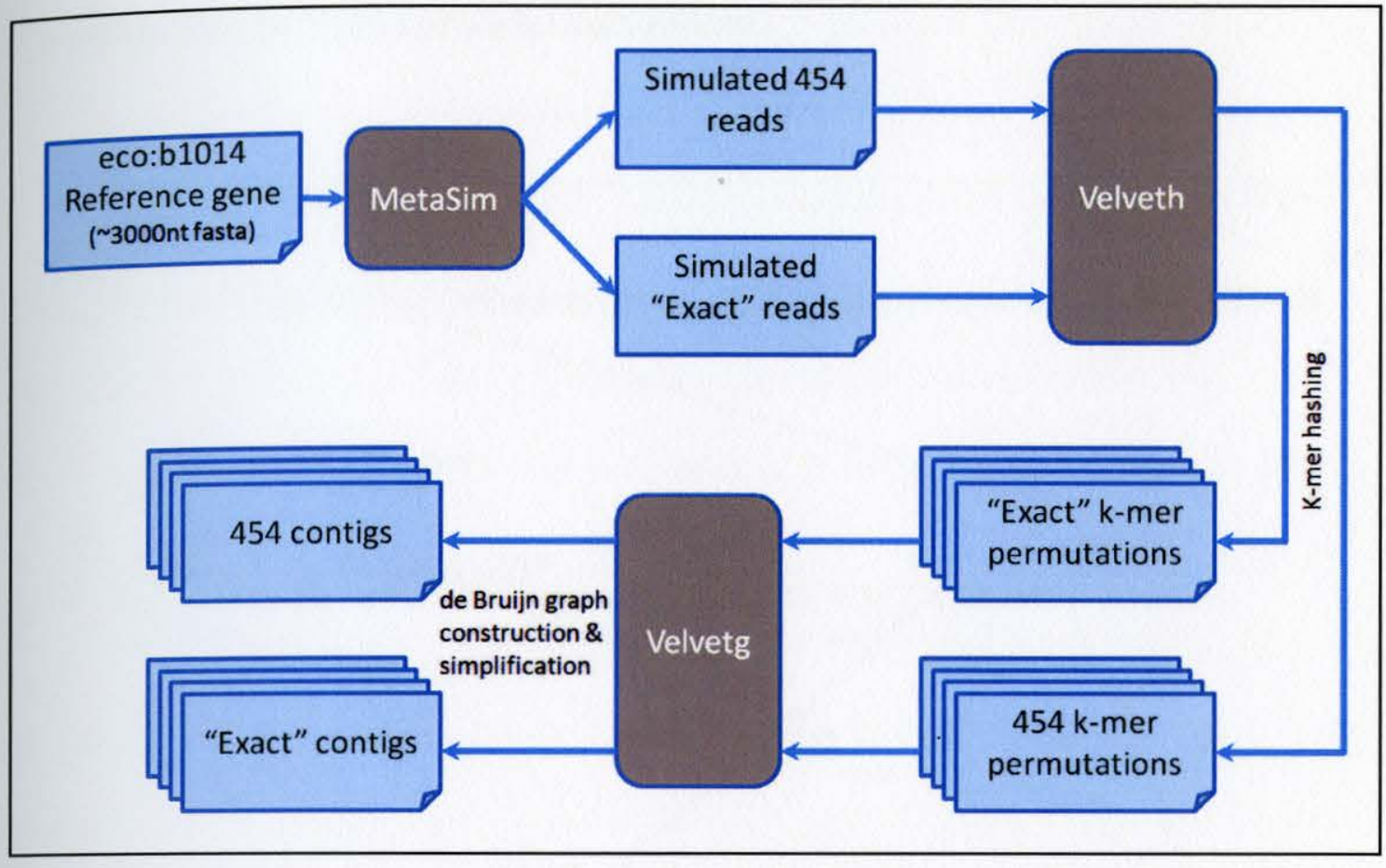

Figure 4: Assembly Programmatic Flow. Metasim generated simulated reads from source gene. These simulated read sets were hashed using velveth with varying k-mer lengths then assembled into de Bruijn graphs using velvetg.

To illustrate the operation of Velvet in conjunction with a series of other utilities (Fig.4), we chose a specific, active coding gene of Escherichia coli str. K12 substr. MG1655 (Fig. 5). This gene, NP_415534, codes for the enzyme proline dehydrogenase/pyrroline-5-carboxylate dehydrogenase which functions as a fused DNA-binding transcriptional regulator (Kyoto University Bioinformatics Center). The E. coli genome has been extensively studied and fully sequenced (Blattner, Plunkett and Bloch) allowing for comparison of our assembly results with established sequence data. The NP_415534 gene sequence was obtained 
from GenomeNet (Kyoto University Bioinformatics Center) in its full form as an ASCII formatted fasta file (NCBI [Fasta]) (Appendix A). This reference gene contains a total of 3963 ordered nucleotides.

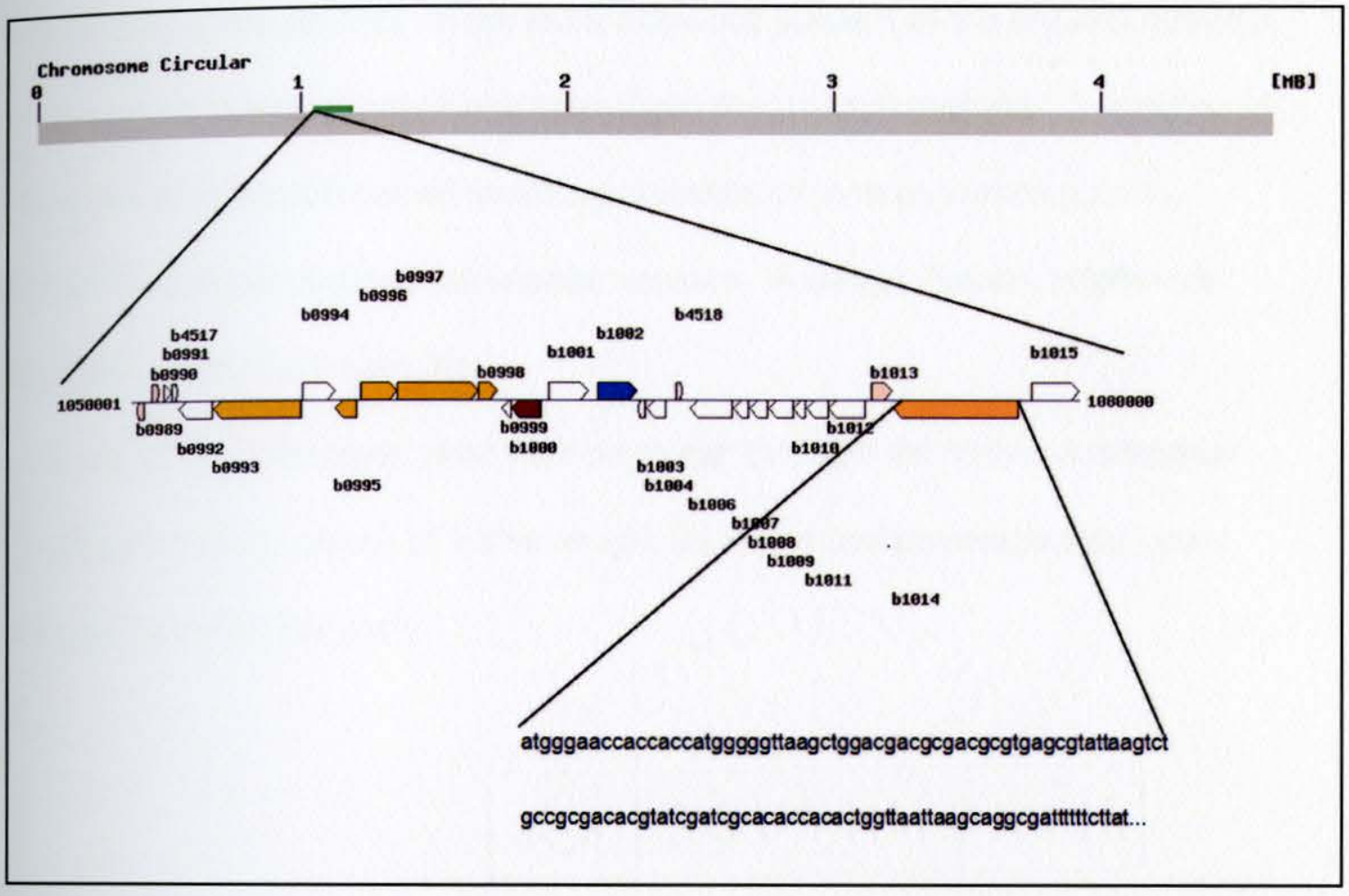

Figure 5: Gene NP_415534 Physical Map. Gene NP_415534, putA was selected from the circular E. Coli chromosome to illustrate the assembly algorithms. This figure shows its physical location with reference to neighboring genes. (Kyoto University Bioinformatics Center)

From the reference file, we used the read simulation function of MetaSim (Richter, et al.) to output two sets of simulated reads (Fig.4). The first set represents an "exact" or reference set (Appendix E) in which, 5000 reads were taken directly from the source gene without introduced error. The output reads have a normal distribution across the source gene and an average read length of 
997.87 base pairs (Appendix E).

To illustrate real world data, we also generated a set of reads modeling the read output of the LifeSciences 454 sequencer (Roche Diagnostics Co.). These 5000 simulated reads contained 29890 insertions and 7321 deletions. Each insertion is the addition of an extra base not present in the original material. Each deletion is the removal of one base from the original material. Locations of these induced errors are based on characteristics of pyrosequencing such difficulties accurately reading homopolar regions. Average Read Length was 258.21 base pairs (Appendix F).

Each of the simulated read sets were run through the Velvet Assembler (Fig.4) using varying values of k-mer length (k), expected coverage (exp_cov) and coverage cutoff (cv_cut). 
Table 1: Assembly Parameter Permutations.

\begin{tabular}{rrrrrrrrrrr}
\hline kmer & cvCut & exp & ctgs & asmLg & N50 & mean & lk & max & tiles & rdPc \\
\hline 21 & 2 & 4 & 4253 & 114471 & 26 & 26 & 0 & 56 & 1394 & 27.88 \\
21 & 3 & 6 & 2603 & 70464 & 26 & 27 & 0 & 44 & 2236 & 44.72 \\
21 & 4 & 8 & 1484 & 40732 & 27 & 27 & 0 & 70 & 2638 & 52.76 \\
21 & 6 & 12 & 107 & 3896 & 36 & 36 & 0 & 144 & 3375 & 67.50 \\
21 & 10 & 20 & 60 & 4007 & 91 & 66 & 0 & 381 & 4997 & 99.94 \\
21 & 12 & 24 & 56 & 4366 & 110 & 77 & 0 & 411 & 5000 & 100.00 \\
23 & 2 & 4 & 4337 & 128149 & 29 & 29 & 0 & 60 & 1378 & 27.56 \\
23 & 3 & 6 & 2638 & 78246 & 28 & 29 & 0 & 66 & 2914 & 58.28 \\
23 & 4 & 8 & 692 & 20845 & 29 & 30 & 0 & 57 & 1438 & 28.76 \\
23 & 6 & 12 & 212 & 7328 & 35 & 34 & 0 & 119 & 3065 & 61.30 \\
23 & 10 & 20 & 65 & 4453 & 99 & 68 & 0 & 311 & 4999 & 99.98 \\
23 & 12 & 24 & 46 & 4099 & 133 & 89 & 0 & 344 & 4998 & 99.96 \\
27 & 2 & 4 & 3989 & 137723 & 33 & 34 & 0 & 82 & 1655 & 33.10 \\
27 & 3 & 6 & 2418 & 83130 & 32 & 34 & 0 & 62 & 1588 & 31.76 \\
27 & 4 & 8 & 1067 & 37296 & 34 & 34 & 0 & 81 & 2490 & 49.80 \\
27 & 6 & 12 & 233 & 9410 & 41 & 40 & 0 & 130 & 3637 & 72.74 \\
27 & 10 & 20 & 47 & 4250 & 136 & 90 & 0 & 559 & 4999 & 99.98 \\
27 & 12 & 24 & 44 & 4331 & 145 & 98 & 0 & 594 & 5000 & 100.00 \\
31 & 2 & 4 & 3804 & 150919 & 38 & 39 & 0 & 81 & 1118 & 22.36 \\
31 & 3 & 6 & 1251 & 49497 & 37 & 39. & 0 & 73 & 596 & 11.92 \\
31 & 4 & 8 & 383 & 15473 & 39 & 40 & 0 & 88 & 1053 & 21.06 \\
31 & 6 & 12 & 134 & 7062 & 54 & 52 & 0 & 195 & 4228 & 84.56 \\
31 & 10 & 20 & 22 & 3605 & 266 & 163 & 0 & 497 & 4975 & 99.50 \\
31 & 12 & 24 & 22 & 3790 & 273 & 172 & 0 & 491 & 5000 & 100.00 \\
\hline & & & & & & & & & &
\end{tabular}

This table shows the parameter permutations used and their results for the simulated 454 reads. "kmer" is the selection of k or kmer length. "cvCut" is coverage cutoff, a threshold used to determine if a node in the constructed de Bruijn graph should be included as part of the final assembly. "exp," expected coverage, is the expected frequency of repeats of each source base. "ctgs" is the number of contigs. "asmLg," "mean," and "max" refer to the total length, mean, and maximum length of all assembled contigs respectively. "N50" refers to the length of the shortest contig in an assembly such that the sum of contigs of equal length or longer is at least $50 \%$ of the total length of all contigs. " 1 " is the number of contigs over 1000 bases long. "tiles" is the number of reads that are used in an assembly. "rdPc" is percentage of input reads used in the assembly.

Automation of parameter variation (Table 1) and report generation (Fig. 6-

9) was assisted by the standardized velvet assembly report script project

(leipzig). Expected coverage is the expected frequency of repeats of each source base. This is a function of the source material and the depth at which the sequencing was performed. Coverage cutoff is the value used to determine if a 
node in the constructed de Bruijn graph should be removed from the final assembly. Lower frequency nodes with coverage below the coverage cutoff value are suspected to be erroneous and are subsequently removed during graph error correction, especially during tip and bulge removal. This threshold specifies how many read k-mers must overlap for each contig k-mer. The number of k-mers per read is a function of read length $L$ and $k$-mer length $K(L-K+1)$ (leipzig). 
Assm. Parameters (kmer/cvCut) vs. Indicators(lgth/N50)

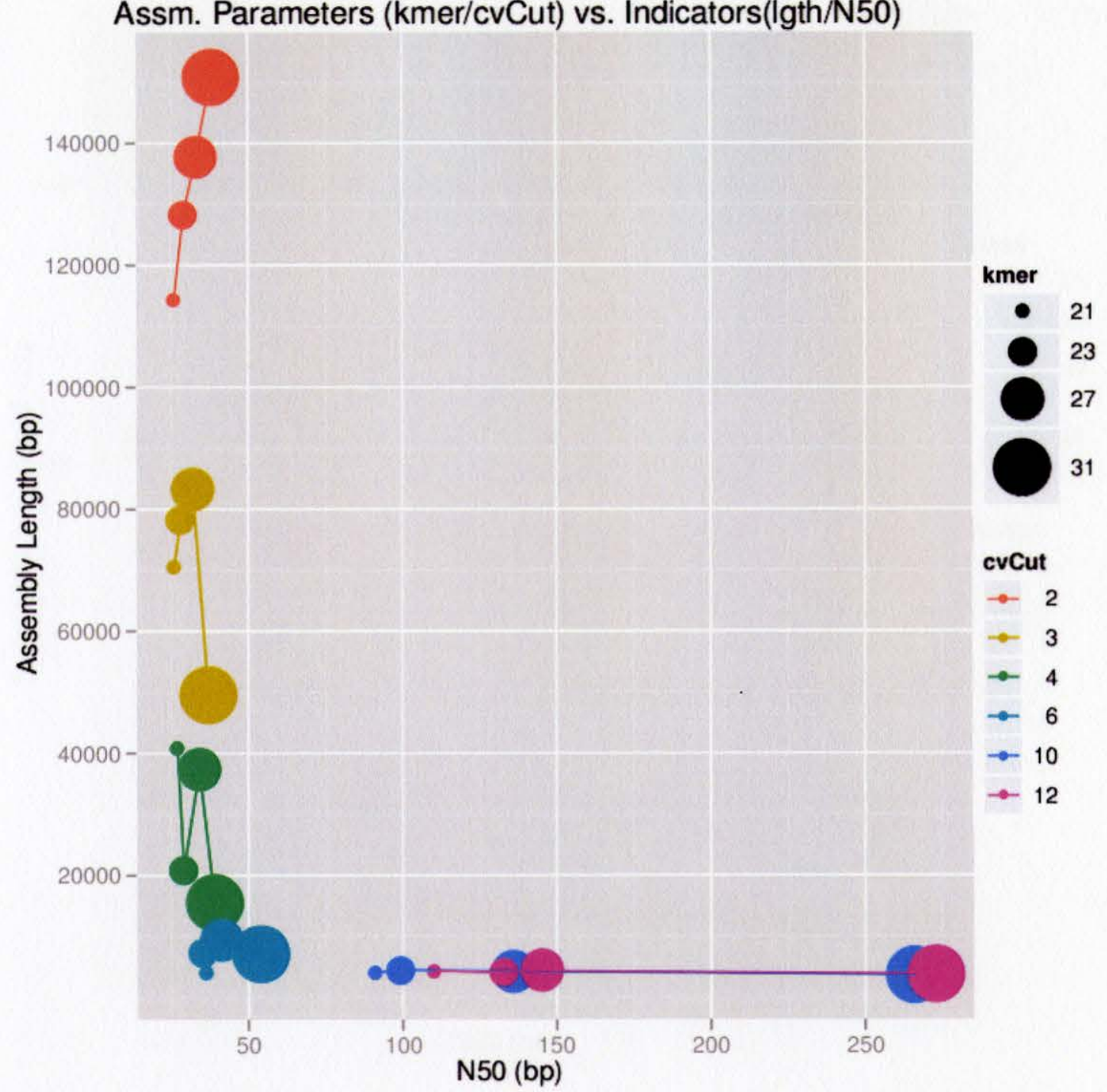

Figure 6: Assembly Parameters (kmer/cvCut) vs. Indicators (lgth/N50). This scatter plot illustrates the effect of kmer length and coverage cutoff on N50 and assembly length. N50 refers to the length of the shortest contig in an assembly such that the sum of contigs of equal length or longer is at least $50 \%$ of the total length of all contigs. Here we see a logarithmic distribution where higher cvCut values generate larger contigs. 
Assm. Parameters (kmer/cvCut) vs. Assm. Indicators(Ctg Count/N50)

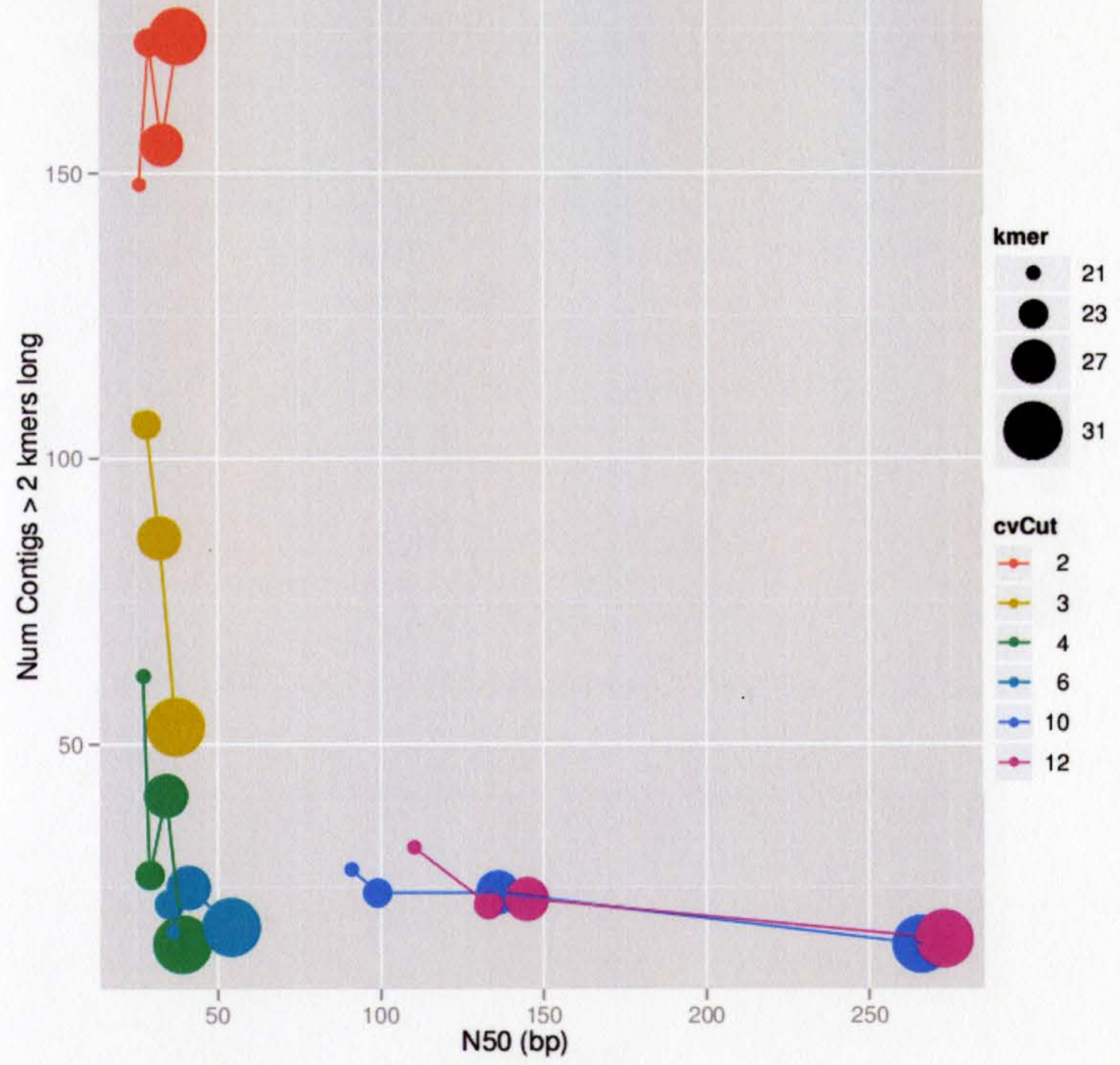

Figure 7: Assembly Parameters (kmer/cvCut) vs. Assembly Indicators (Ctg Count/N50). This plot shows the influence of kmer length and cvCut on the number of contigs produced with greater than $2^{*} k$ length. Again, we see a somewhat logarithmic function with higher cvCut and higher kmers producer longer and fewer isolated contigs. 
Assm. Parameters (kmer/cvCut) vs. Percent Read Usage

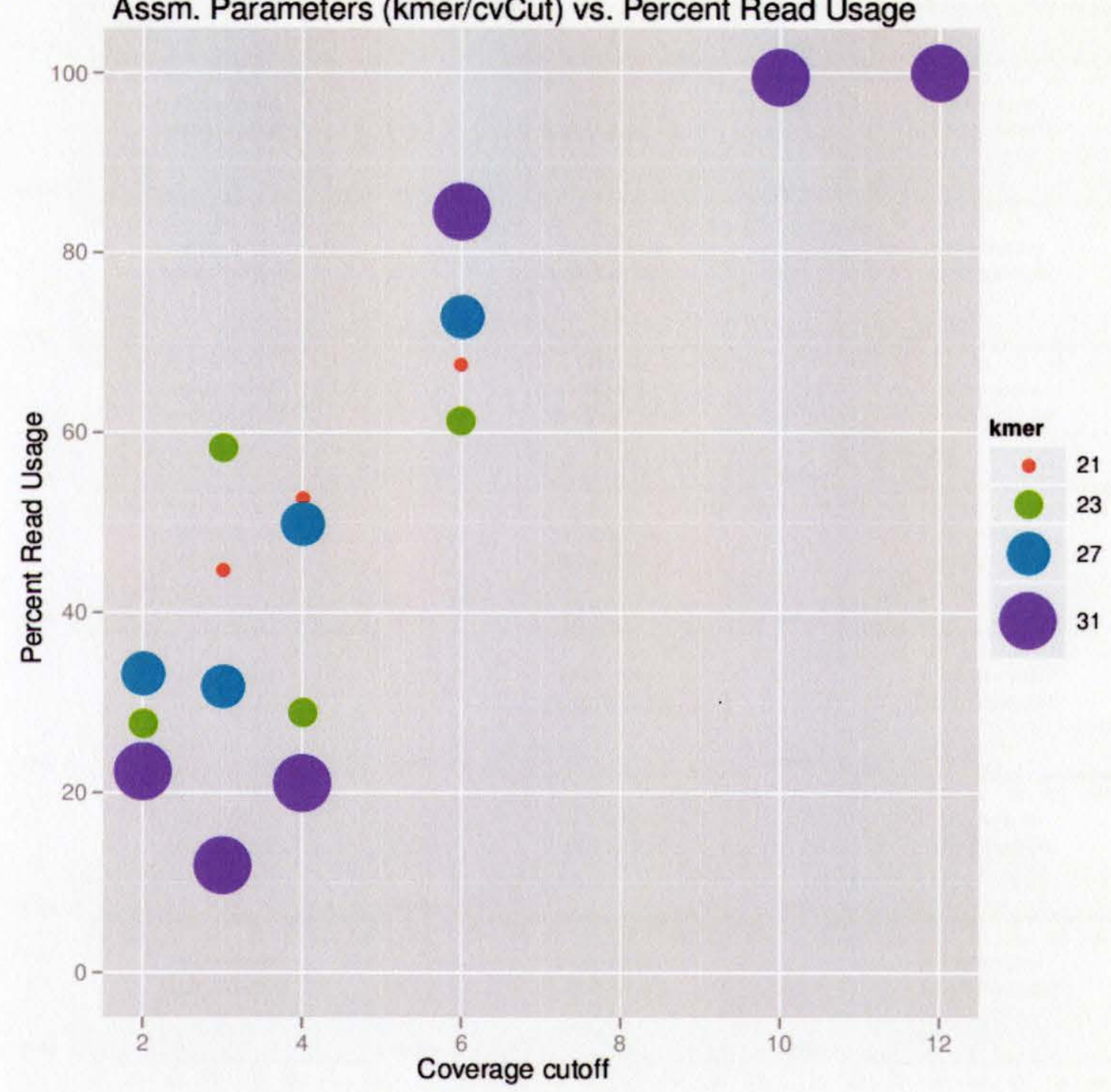

Figure 8: Assembly Parameters (k-mer/cvCut) vs. Percent Read Usage. This figure compares k-mer and cvCut to the percent read usage. With sufficiently high $\mathrm{k}$, read utilization increases with coverage cutoff, due to the relaxation of selectivity of node removal. 


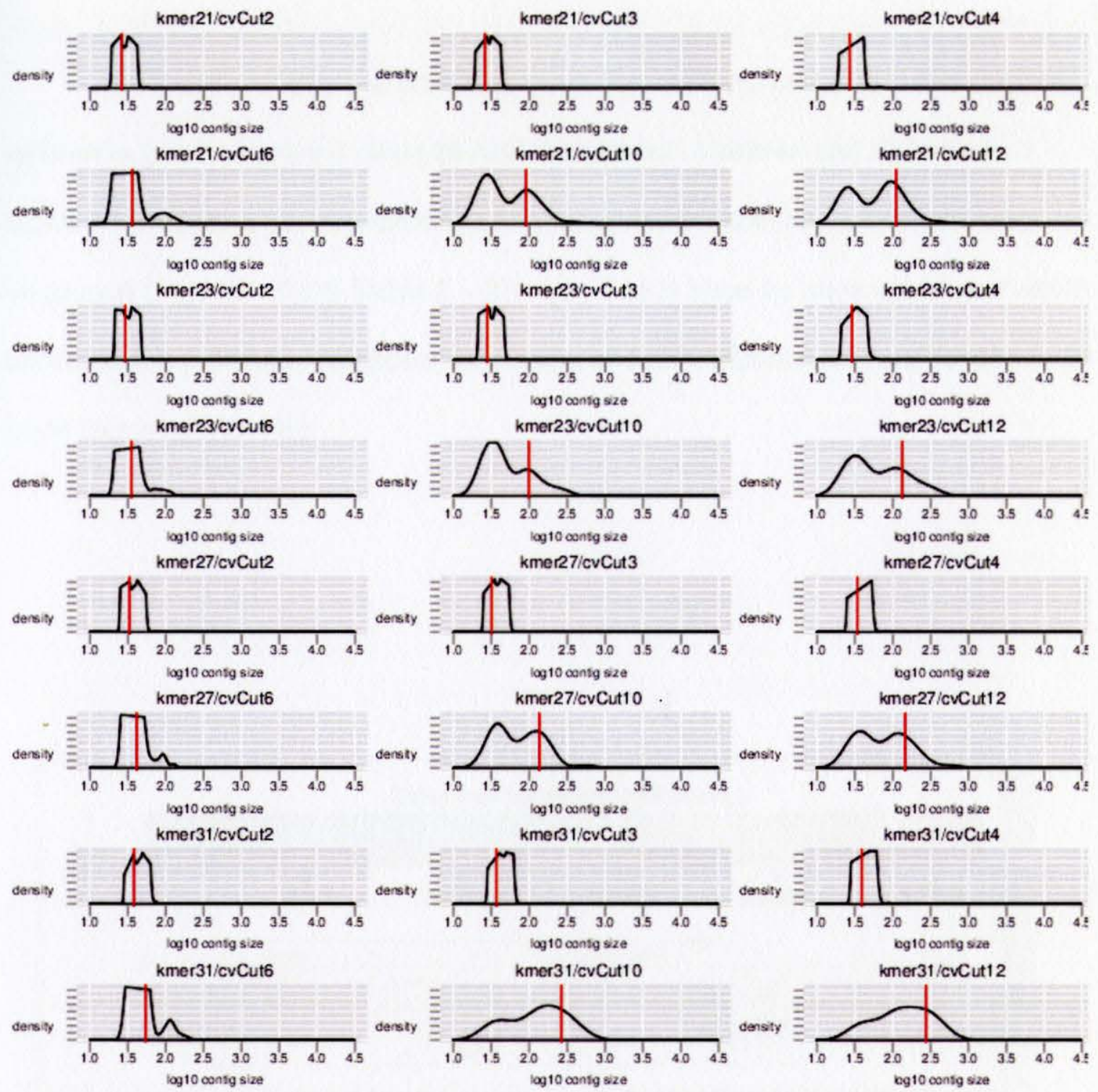

Figure 9: Kernel density plot of log contig size distribution by controlling for overall contig count. It is interesting to note the bimodal nature of this data. Future research into the cause of this may uncover underlying information about the source data or function of the assembler. The highest kmer and cvCut runs appear to be more normal in distribution. 
The results of these assemblies were then compared back to the original, reference gene sequence using BLASTN (Altschul, Madden and Schaffer).

BLASTN outputs a percent identity which shows the similarity to the reference sequence (Fig. $10-12 \&$ Table $2-3$ ) as well as a base by base alignment which shows direct matches, deletions, insertions and substitutions for each assembled node (Appendix B - E).

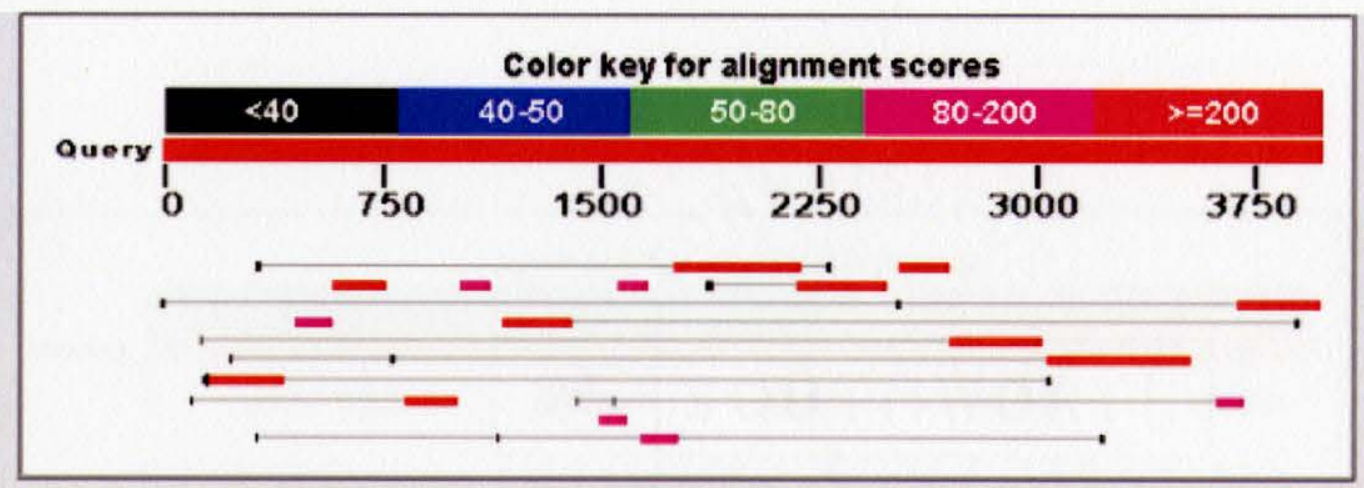

Figure 10: BLAST Map for Simulated 454 Reads $(k=31$, coverage cutoff $=12$, expected coverage $=24$ ). This figure, from BLAST, maps the 16 resultant contigs of the 454 simulated reads at k-mer length 31 , expected coverage 24 , and coverage cutoff 12 . The level of similarity to the reference gene is shown by the color, with red being the best quality. Contigs appear to high quality and well distributed about the gene. 
Table 2: BLAST Contig Scoring for Simulated 454 Reads $(k=31$, coverage cutoff $=12$, expected coverage $=24$ )

\begin{tabular}{|c|c|c|c|c|c|c|}
\hline Accession & Description & Hax seore & Total score & Query coverage & $\angle$ Evalue & Maxident \\
\hline 63088 & NODE_1_length_393_cov_219.491089 & $\underline{623}$ & 713 & $11 \%$ & 0.0 & $100 \%$ \\
\hline 63089 & NODE_2 length_282_cov_195.992905 & $\underline{484}$ & 511 & $8 \%$ & $2 e-140$ & $96 \%$ \\
\hline 63090 & NODE_3 Jength_267_cov_206.041199 & 428 & 479 & $7 \%$ & $1 e-123$ & $100 \%$ \\
\hline 63091 & NODE_4_length_206_cov_205.684464 & $\underline{340}$ & 361 & $6 \%$ & $3 e-97$ & $100 \%$ \\
\hline 63092 & NODE_5_length_158_cov_201.348099 & $\underline{293}$ & 293 & $4 \%$ & $3 e-83$ & $95 \%$ \\
\hline 63093 & NODE_6 length_291_cov_199.041245 & $\underline{524}$ & 570 & $8 \%$ & $2 e-152$ & $100 \%$ \\
\hline 63094 & NODE_7_length_461_cov_209.563995 & $\underline{690}$ & 732 & $13 \%$ & 0.0 & $100 \%$ \\
\hline 63095 & NODE_8_length_243_cov_213.312759 & $\underline{416}$ & 458 & $7 \%$ & $6 e-120$ & $100 \%$ \\
\hline 63096 & NODE_9 length_98_cov_212.948975 & 197 & 197 & $3 \%$ & $1 e-54$ & $96 \%$ \\
\hline 63097 & NODE_10_length_148_cov_229.418915 & $\underline{300}$ & 321 & $4 \%$ & $2 e-85$ & $100 \%$ \\
\hline 63098 & NODE_11_length_76_cov_221.750000 & $\underline{156}$ & 156 & $2 \%$ & $3 e-42$ & $95 \%$ \\
\hline 63099 & NODE_12 length_62_cov_208.145157 & 129 & 171 & $2 \%$ & $4 e-34$ & $100 \%$ \\
\hline 63100 & NODE_13_length_73_cov_221.095886 & $\underline{187}$ & 187 & $2 \%$ & $2 e-51$ & $100 \%$ \\
\hline 63101 & NODE_15_length_142_Cov_198.697189 & $\underline{239}$ & 239 & $4 \%$ & $6 e-67$ & $92 \%$ \\
\hline 63102 & NODE_ 16 length_65_cov_216.415390 & 114 & 114 & $2 \%$ & $8 e-30$ & $88 \%$ \\
\hline 63103 & NODE 17 length 101 cov 213.732666 & $\underline{185}$ & 252 & $4 \%$ & $8 e-51$ & $100 \%$ \\
\hline
\end{tabular}

This table, also from BLAST, shows the 16 resultant contigs of the 454 simulated reads at $k$-mer length 31 , coverage cutoff 12 and expected coverage 24 . The contigs averaged $97.63 \%$ accuracy.

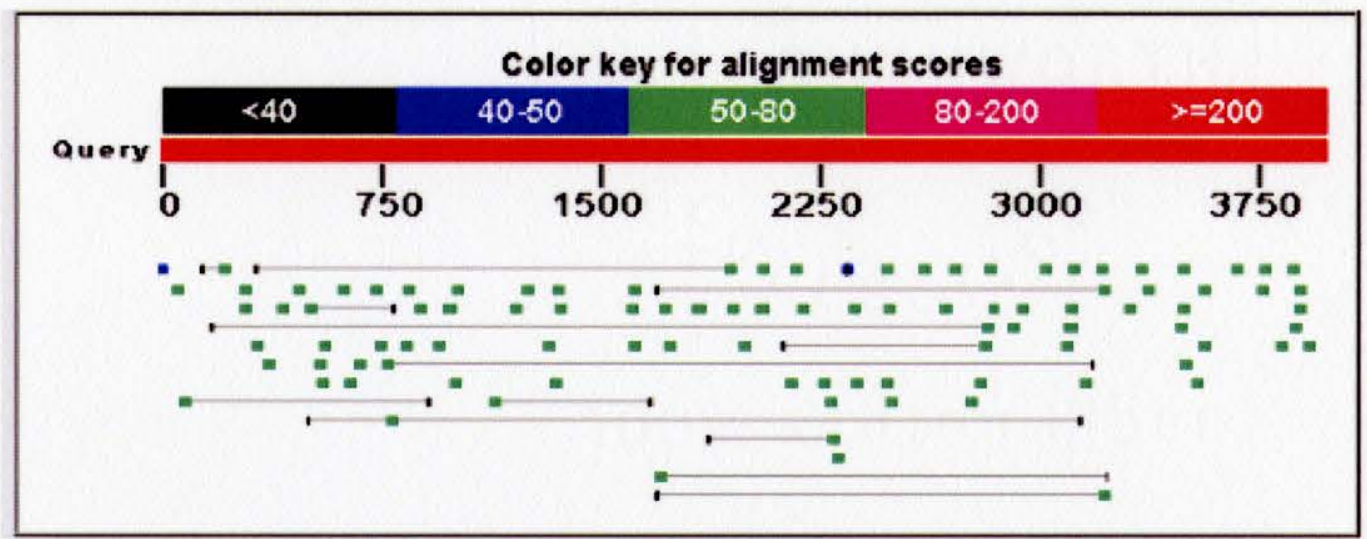

Figure 11: BLAST Contig Scoring for Simulated 454 Reads $(k=21$, coverage cutoff $=2$, expected coverage $=4$ ). This assembly resulted in 4253 nodes and $n 50$ of 16 . BLAST, maps the resultant contigs above a scoring threshold. The level of similarity to the reference gene is shown by the color, with red being the best quality. Contigs appear to be very small with medium to poor quality, yet well distributed about the gene. 
Table 3: BLAST Contig Scoring for Simulated 454 Reads $(k=21$, coverage cutoff $=2$, expected coverage $=4$ )

\begin{tabular}{|c|c|c|c|c|c|c|c|}
\hline Accession & Description & Mexacere & Total exors & Query coverage & Evertun & Maxident & Lnks \\
\hline 52974 & NODE 11 length 21 cov 7.142857 & 662.2 & 87.3 & $1 \%$ & $1 e \cdot 15$ & $100 \%$ & \\
\hline 52975 & NODE_ 159 sength 21 cov_2.000000 & 59.0 & 59.0 & $0 \%$ & $2 e-13$ & $95 \%$ & \\
\hline 52976 & NODE 273 Jength 21 cov 3.952381 & 65.2 & 66.2 & $1 \%$ & 1e-15 & $97 \%$ & \\
\hline 52977 & NOOE_893_Jength_21_cov_5.047619 & 65.2 & 92.7 & $1 \%$ & 1e-15 & $97 \%$ & \\
\hline 52978 & NODE_1046_length_21_cov_6.666667 & 65.2 & 66.2 & $1 \%$ & 1e-15 & $97 \%$ & \\
\hline 52979 & NOOE_1086 Jength_23_COv_93.173912 & 53.6 & 53.6 & $1 \%$ & $9 e \cdot 12$ & 93\% & \\
\hline 52980 & NOOE_ 1139 length_21_cov_27.714285 & 45.4 & 46.4 & o\% & $1 e-09$ & $96 \%$ & \\
\hline 52981 & NOOE_ 1229 Jength_21_COV_ 3.571429 & 65.2 & 66.2 & $1 \%$ & $1 e \cdot 15$ & $97 \%$ & \\
\hline 52982 & NODE 1278 jength 21 Cov_ 4.047619 & 65.2 & 66.2 & $1 \%$ & $1 e \cdot 15$ & $97 \%$ & \\
\hline 52983 & NOOE_ 1407 length_21_cov_ 4.904762 & $\$ 6.2$ & 66.2 & $1 \%$ & $1 e-15$ & $97 \%$ & \\
\hline 52984 & NOOE_1540_Jength_36_cov_27.888389 & 42.8 & 83.7 & $0 \%$ & 20.08 & $100 \%$ & \\
\hline 52985 & NODE_ 1819 _length_25_cov_2.360000 & 590 & 81.9 & $1 \%$ & 2e-13 & $93 \%$ & \\
\hline 52986 & NOOE 1834 lenguh 21 COV 5.523809 & 66.2 & 66.2 & $1 \%$ & 1e-15 & $97 \%$ & \\
\hline 52987 & NODE 1931 Jength 21 Cov 4.904762 & 65.2 & 92.7 & $1 \%$ & $1 e-15$ & $100 \%$ & \\
\hline 52988 & NODE 1961 Jength 21 cav 2.428571 & 66.2 & 66.2 & 136 & $1 e-15$ & $97 \%$ & \\
\hline 52989 & NODE 1994 length 21 cov 5.000000 & 65.2 & 87.3 & $1 \%$ & $1 e-15$ & $100 \%$ & \\
\hline 52990 & NOOE_ 2005 Jength_21_cov_ 4.714286 & 65.2 & 66.2 & $1 \%$ & $1 e-15$ & $97 \%$ & \\
\hline 52991 & NOOE_2082 Jength_21_cov_3.238095 & 66.2 & 66.2 & $1 \%$ & 1e-15 & $97 \%$ & \\
\hline 52992 & NODE 2191 length 21 cov_ 5.095238 & 66.2 & 66.2 & $1 \%$ & $1 e-15$ & $97 \%$ & \\
\hline 52993 & NOOE, 2199 length 21 cov_ 7.380952 & 56.2 & 66.2 & $1 \%$ & $1 e-15$ & $97 \%$ & \\
\hline 52994 & NODE 2212 Jength 21 cov 4.238095 & 66.2 & 66.2 & $1 \%$ & $1 e-15$ & $97 \%$ & \\
\hline 52995 & NODE 2217 length 21 cov_5.904762 & 66.2 & 66.2 & $1 \%$ & $1 e-15$ & $97 \%$ & \\
\hline 52996 & NODE_2236_Jength_21_cov_6.190476 & 662 & 66.2 & $1 \%$ & $1 e-15$ & $97 \%$ & \\
\hline 52997 & NODE_2230 Jength_21_cov_2.857143 & 66.2 & 66.2 & $1 \%$ & $1 \mathrm{e}-15$ & $97 \%$ & \\
\hline 52998 & NODE 2257 Jength 21 cov_ 4.714286 & 66.2 & 66.2 & $1 \%$ & $1 \mathrm{e}-15$ & $97 \%$ & \\
\hline 52999 & NOOE_ 2258 _length_21_cov_ 4.523809 & 66.2 & 66.2 & $1 \%$ & $1 e-15$ & 9796 & \\
\hline 53000 & NODE 2275 length 21 cov 3.095238 & $\$ 5.2$ & 66.2 & $1 \%$ & $1 e-15$ & $97 \%$ & \\
\hline 53001 & NODE 2294 Jength 21 cav 3.428571 & 65.2 & 66.2 & $1 \%$ & $1 e-15$ & $97 \%$ & \\
\hline 53002 & NODE_2316_length_21_cov_4.714286 & 66.2 & 66.2 & $1 \%$ & $1 e-15$ & $97 \%$ & \\
\hline 53003 & NODE 2332 length 21 cov 2.619048 & 6.2 & 66.2 & $1 \%$ & $1 \mathrm{e}-15$ & $97 \%$ & \\
\hline 53004 & NODE 2338 jength 21 cov_ 4.857143 & 66.2 & 66.2 & $1 \%$ & $1 e-15$ & $97 \%$ & \\
\hline 53005 & NODE 2351 length 21 cov_ 4.761905 & 66.2 & 66.2 & $1 \%$ & $1 e \cdot 15$ & $97 \%$ & \\
\hline 53006 & NODE 2355 length 21 cov_ 7.142857 & 66.2 & 66.2 & $1 \%$ & $1 e-15$ & $97 \%$ & \\
\hline 53007 & NODE_2369 Jength 21 cov_ 3.428571 & 652 & 66.2 & $1 \%$ & $1 e-15$ & $97 \%$ & \\
\hline 53008 & NODE_2370_length_21_cov_7.190476 & 65.2 & 66.2 & $1 \%$ & $1 e-15$ & $97 \%$ & \\
\hline 53009 & NODE_2371 Jength_21_Cov_4.190476 & 66.2 & 66.2 & $1 \%$ & $1 e-15$ & $97 \%$ & \\
\hline
\end{tabular}


Color key for alignment scores

\begin{tabular}{c|crrrrr} 
& $<40$ & & $40-50$ & $50-80$ & $80-200$ & $>=200$ \\
\hline \multirow{2}{*}{ Query } & & 750 & 1500 & 2250 & 3000 & 3750
\end{tabular}

Figure 12: BLAST Contig Scoring for Simulated "Exact" Reads $(\mathrm{k}=$ 21 , coverage cutoff $=2$, expected coverage $=4$ ). This assembly maps the single resultant contig of the simulated "exact" reads at k-mer length 21, expected coverage 4 , and coverage cutoff 2 . This assembly resulted in a complete gene sequence regardless of input parameters, indicating that the importance of coverage cutoff and expected coverage lies mainly in error handling and correction. When the input was of complete consensus with the reference sequence, the output remained error free. The assembled contig achieved $100 \%$ reference gene coverage at $100 \%$ identity with a length of 3963 bases.

AMOS files of selected final assemblies were generated with velvet and opened for analysis with Hawkeye (Fig. 13-15) (Schatz, Phillippy and Shneiderman). 


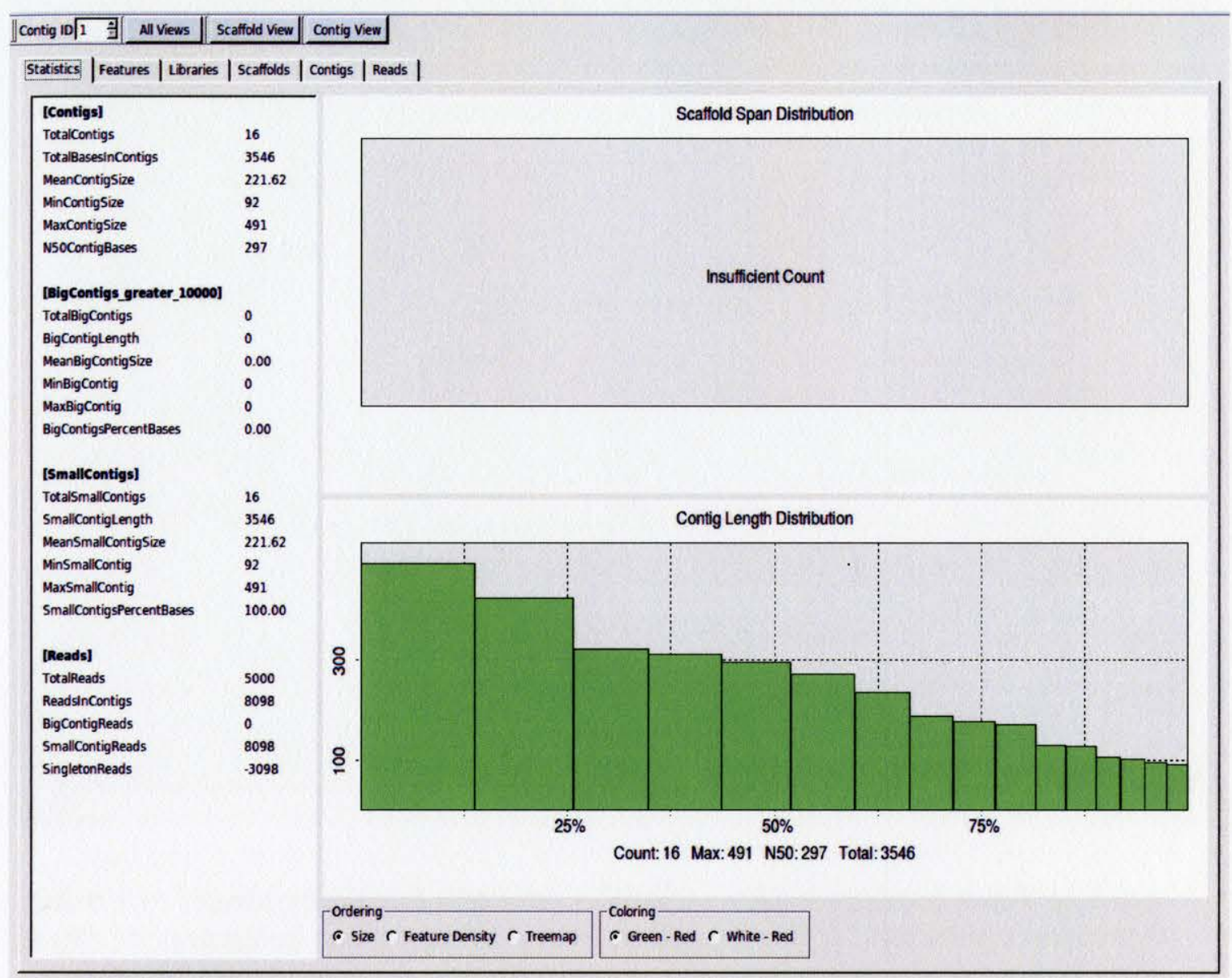

Viewing 454_31_12_24.bnk with 16 contigs Contig Id:1 Size: 423 Reads: 775

Figure 13: Hawkeye overview display. Simulated 454 simulated reads at k-mer length 31 , expected coverage 24 , and coverage cutoff 12 . Various statistics are shown, including a graph of the contig length distribution. 


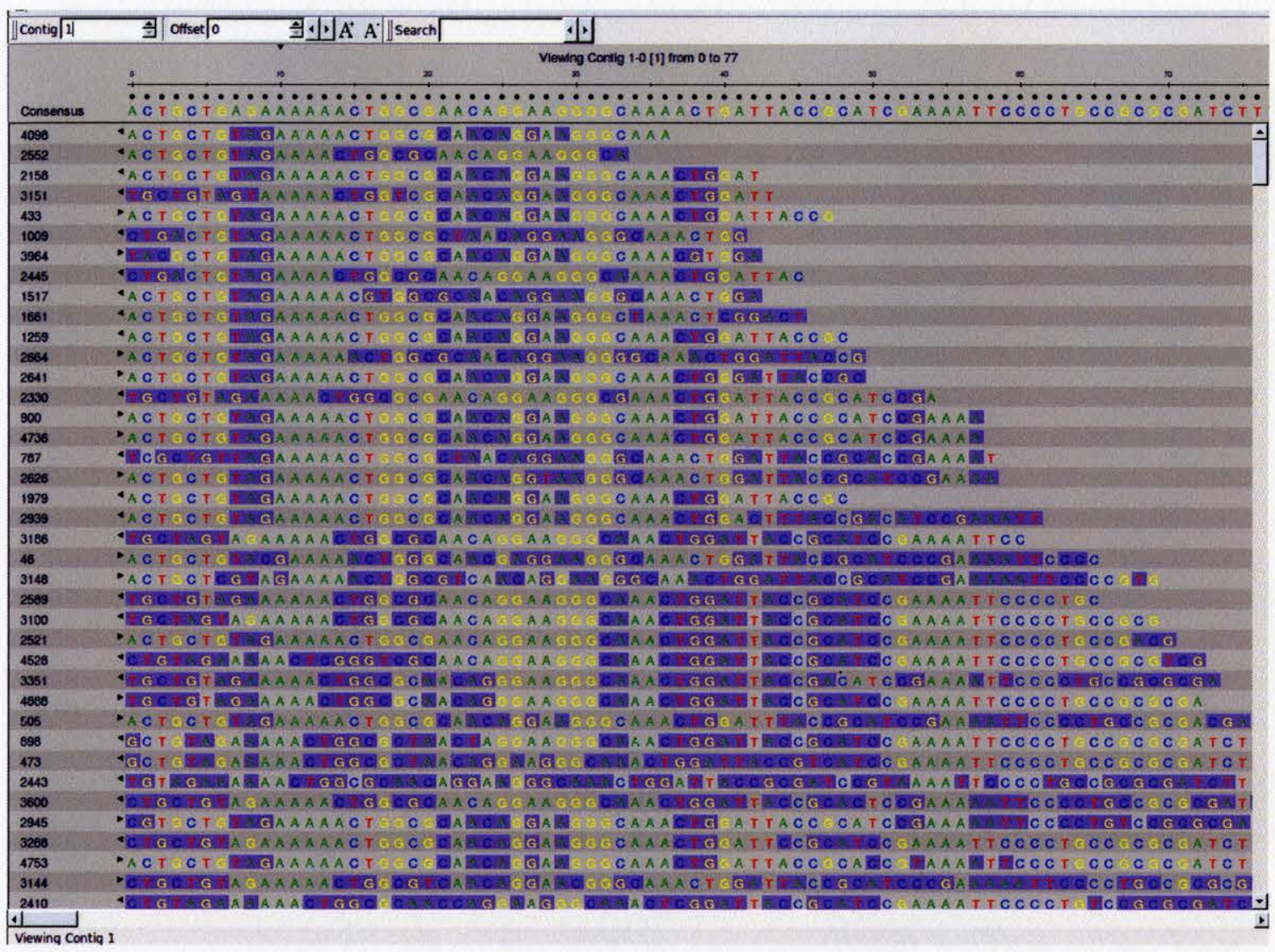

Figure 14: Hawkeye Contig Alignment Display. 454 simulated reads at k-mer length 31 , expected coverage 24 , and coverage cutoff 12 showing consensus alignments of the reads used to create one of the 16 generated contigs. This figure also shows the introduced errors in the simulated reads. 


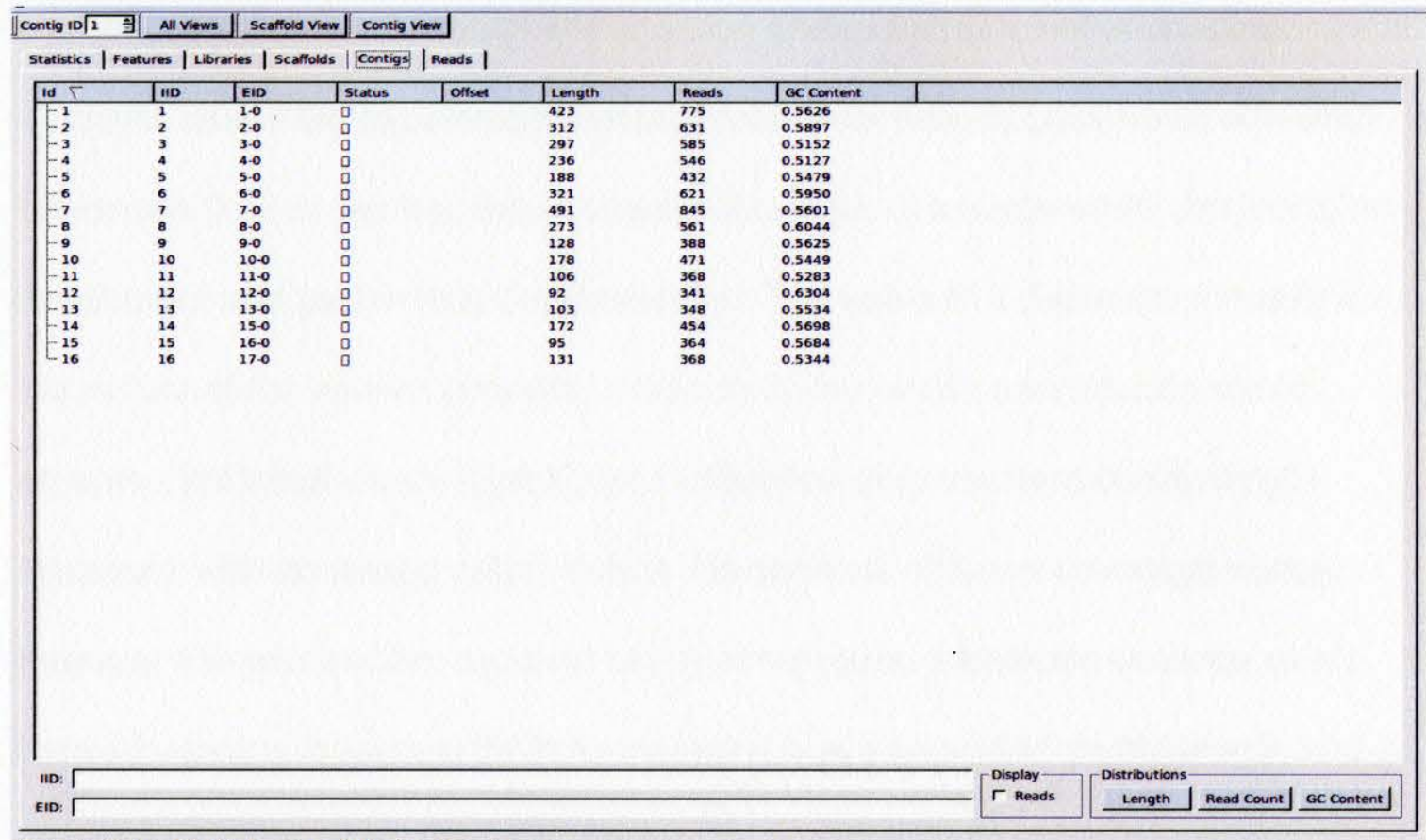

Viewinn 454 91 12,24 hak with 16 rontins Cnntin Id:1 Sige: 427 Reards: 775

Figure 15: Hawkeye Contig Detail Display. 454 simulated reads at k-mer length 31 , expected coverage 24 , and coverage cutoff 12 showing information about each of the 16 generated contigs including length and number of reads used per contig. 


\section{CHAPTER IV}

\section{CONCLUSIONS}

Read assembly remains an inexact science, relying heavily on statistical modeling and inference for error correction and graph simplification. Our synthetic assembly experiment demonstrates how heavily parameter selection influences final assembly, thus consideration must be made when designing an experiment and performing the assembly. The value of $k$ depends primarily on the nature of the source genome, particularly the length and abundance of repeats. With sufficiently high $\mathrm{k}$, read utilization and resultant contig length increases with coverage cutoff, due to the removal of lower coverage nodes, however this elimination can lead to misassemblies. A delicate balance exists between easing coverage limits to increase final assembled contig length and a reduction in accuracy. Some experiments, such as preliminary genome sequencing may seek wider coverage and fewer but longer nodes at the expense of $100 \%$ accuracy of individual bases, whereas small target sequencing of short gene segments may obtain the higher accuracy required by increasing read coverage.

\section{Future Work}

As the algorithms continue to mature, research into the automated choice of parameters will assist scientists when faced with the challenge of read assembly. Obtaining and integrating the various scripts and applications was a chore, as each had its own set of dependencies and special setup instructions. 
Velvet assembly and the associated tools would benefit from a cloud implementation, similar to that of NCBI's BLAST to provide a full suite of assembly tools with minimal or no configuration.

Further efforts to understand the parameterization of short read assembly using Velvet should expand both the source data and selected parameter value set, possibly to include eukaryotic data. Application of a similar study to eukaryotic source data could aid in understanding how the various stages of De Novo assembly are influenced by the fundamental differences in complexity and arrangement of more complex genomes. Understanding the effects that source data repeat rates and complexity have on assembly contig outputs may lead to a better understanding or expansion of the use boundaries of this algorithm.

A more detailed study of k-mer length selection could also include recursive scanning of a reference genome for maximum repeat length and a priori comparison to the genomes of similar organisms.

Continued effort to understand and evaluate the decisions used when simplifying or error correcting the de Bruijn graph will lead to higher quality assemblies, eventually reducing our dependence on De Novo techniques. Improved and standardized methods can server to unify the field in the areas of statistical decision making as well as reference to biological markers and archived genomic data. 


\section{APPENDIX A}

\section{FASTA FILE FOR REFERENCE GENE PUTA/B1014}

>eco:b1014 putA, ECK1005, JW0999, poaA, putC; fused DNA-binding transcriptional regulator/proline dehydrogenase/pyrroline-5-carboxylate dehydrogenase (EC:1.5.99.8 1.5.1.12); K00294 1-pyrroline-5-carboxylate dehydrogenase [EC:1.5.1.12] K00318 proline dehydrogenase [EC:1.5.99.8] (N) atgggaaccaccaccatgggggttaagctggacgacgcgacgcgtgagcgtattaagtct gccgcgacacgtatcgatcgcacaccacactggttaattaagcaggcgatttttcttat ctcgaacaactggaaaacagcgatactctgccggagctacctgcgctgctttctggcgcg gccaatgagagcgatgaagcaccgactccggcagaggaaccacaccagccattcctcgac tttgccgagcaaatattgccccagtcggtttcccgcgccgcgatcaccgcggcctatcgc cgcccggaaaccgaagcggtttctatgctgctggaacaagcccgcctgccgcagccagtt gctgaacaggcgcacaaactggcgtatcagctggccgataaactgcgtaatcaaaaaaat gccagtggtcgcgcaggtatggtccaggggttattgcaggagtttcgctgtcatcgcag gaaggcgtggcgctgatgtgtctggcggaagcgttgttgcgtattcccgacaaagccacc cgcgacgcgttaattcgcgacaaaatcagcaacggtaactggcagtcacacattggtcgt agcccgtcactgtttgttaatgccgccacctgggggctgctgtttactggcaaactggtt tccacccataacgaagccagcctctcccgctcgctgaaccgcattatcggtaaaagcggt gaaccgctgatccgcaaaggtgtggatatggcgatgcgcctgatgggtgagcagttcgtc actggcgaaaccatcgcggaagcgttagccaatgcccgcaagctggaagagaaaggtttc cgttactcttacgatatgctgggcgaagccgcgctgaccgccgcagatgcacaggcgtat atggtttcctatcagcaggcgattcacgccatcggtaaagcgtctaacggtcgtggcatc tatgaagggccgggcatttcaatcaaactgtcggcgctgcatccgcgttatagccgcgcc cagtatgaccgggtaatggaagagctttacccgcgtctgaaatcactcaccctgctggcg cgtcagtacgatattggtatcaacattgacgccgaagagtccgatcgcctggagatctcc ctcgatctgctggaaaaactctgtttcgagccggaactggcaggctggaacggcatcggt tttgttattcaggcttatcaaaaacgctgcccgttggtgatcgattacctgattgatctc gccacccgcagccgtcgccgtctgatgattcgcctggtgaaaggcgcgtactgggatagt gaaattaagcgtgcgcagatggacggccttgaaggttatccggtttatacccgcaaggtg tataccgacgttcttatctcgcctgtgcgaaaaagctgctggcggtgccgaatctaatc tacccgcagttcgcgacgcacaacgcccatacgctggcggcgatttatcaactggcgggg cagaactactacccgggtcagtacgagttccagtgcctgcatggtatgggcgagccactg tatgagcaggtcaccgggaaagttgccgacggcaaacttaaccgtccgtgtcgtatttat gctccggttggcacacatgaaacgctgttggcgtatctggtgcgtcgcctgctggaaaac ggtgctaacacctcgtttgttaaccgtattgccgacacctcttgccactggatgaactg gtcgccgatccggtcactgctgtagaaaaactggcgcaacaggaagggcaaactggatta ccgcatccgaaaattccctgccgcgcgatctttacggtcacgggcgcgacaactcggca gggctggatctcgctaacgaacaccgcctggcctcgctctcctctgccctgctcaatagt gcactgcaaaaatggcaggccttgccaatgctggaacaaccggtagcggcaggtgagatg tcgcccgttattaaccctgcggaaccgaaagatattgtgggetatgtgcgtgaagccacg ccgcgtgaagtagaacaggcgctggaaagtgcggttaataacgcgccaatctggtttgcc acgcctccggctgaacgcgcagcgatttgcaccgcgctgccgtgctgatggaaagccag atgcagcaactgattggtattctggtgcgtgaggccggaaaaaccttcagtaacgccatt gccgaagtgcgcgaagcggtcgattttctccactactacgccggacaggtgcgggatgat 
ttcgctaacgaaacccaccgtccattagggcctgtggtgtgtatcagtccgtggaacttc ccgctggctattttcaccgggcagatcgccgccgcactggcggcaggtaacagcgtgctg gcaaaaccggcagaacaaacgccgctgattgccgcgcaagggatcgccatttgctggaa gcgggtgtaccgccaggcgtggtgcaattgctgccaggtcggggtgaaaccgtgggcgcg caactgacgggtgatgatcgcgtgcgcggggtgatgtttaccggttcaaccgaagtcgct acgttactgcagcgcaatatcgccagccgcctggacgctcagggtcgccctattccgctc atcgctgaaaccggcggcatgaacgcgatgattgtcgattcttcagcactgaccgaacag gtcgtcgtggatgtactggcctcggcgttcgacagtgcgggtcagcgttgttcggcgctg cgcgtgctgtgcctgcaagatgagattgccgaccacacgttgaaaatgctgcgcggcgca atggccgaatgccggatgggtaatccgggtcgectgaccaccgatatcggtccagtgatt gatagcgaagcgaaagccaatattgagcgccatattcagaccatgcgtagcaaaggccgt ccggtgttccaggcggtgcgggaaaacagcgaagatgcccgtgaatggcaaagcggcacc ttgtcgccccgacgctgatcgaactggatgactttgccgaattgcaaaaagaggtcttt ggtccggtgctgcatgtggtgcgttacaaccgtaaccagctaccagagctgatcgagcag attaacgcttccggttatggtctgacgcttggcgtccatacgcgcattgatgaaaccatc gcccaggtcactggctcggcccatgttggtaacctgtatgttaaccgtaatatggtgggc gcagtggttggtgtgcagccgttcggcggcgaagggttgtccggtaccgggccgaaagca ggcggtccgctctatctctaccgtctgctggcgaatcgcccggaaagtgcgctggcagtg acgctcgcgcgtcaggatgcaaagtatccggtcgatgcgcagttgaaagccgcattgact cagccgctaaatgcactgcgggaatgggcagcaaatcgtccagaattgcaggcgttatgt acgcaatatggcgagctggcgcaggcaggaacacaacgattgctgccggggccgacgggt gaacgcaacacctggacgctgctgccgcgtgagcgcgtgttgtgtattgccgatgatgag caggatgcgctgactcagctcgccgccgtgctggcggtgggcagccaggtactgtggccg gatgacgcgctgcatcgtcagttagtgaaggcattgccatcggcagtcagcgaacgtatt caactggcgaaagcggaaaatataaccgctcaaccgtttgatgcggtgatcttccacggt gattcggatcagcttcgcgcattgtgtgaagcagttgccgcgcgggatggcacaattgtt tcggtgcagggtttgcccgtggcgaaagcaatatccttctggaacggctgtatatcgag cgttcgctgagtgtgaataccgctgccgctggcggtaacgccagcttaatgactataggt taa 


\title{
APPENDIX B
}

\section{BLAST REPORT "EXACT" ASSEMBLED $k=21$ EXPECTED COVERAGE $=4$ \\ COVERAGE CUTOFF $=2$ VS. REFERENCE}

\begin{abstract}
velveth out_NP_415534-Exact_21 21 -fasta -shortPaired NP_415534-Exact.fasta velvetg out_NP_415534-Exact_21_2_4_dir -exp_cov 4 -cov_cutoff 2 -read_trkg yes amos_file yes -unused_reads yes

Final graph has 1 nodes and 550 of 3963, max 3963, total 3963, using 5000/5000 reads
\end{abstract}

BLASTN 2.2.23+

Reference: Stephen F. Altschul, Thomas L. Madden, Alejandro

A. Schaffer, Jinghui Zhang, Zheng Zhang, Webb Miller, and

David J. Lipman (1997), "Gapped BLAST and PSI-BLAST: a new

generation of protein database search programs", Nucleic

Acids Res. 25:3389-3402.

RID: U317VMZJ112

Query = eco:b1014 putA, ECK1005, JW0999, poaA, putC; fused DNA-binding transcriptional regulator/proline dehydrogenase/pyrroline-5-carboxylate dehydrogenase (EC:1.5.99.8

1.5.1.12); K00294 1-pyrroline-5-carboxylate dehydrogenase [EC:1.5.1.12] K00318 proline dehydrogenase [EC:1.5.99.8] (N) Length $=3963$

Sequences producing significant alignments:

1c1|29253 NODE_1_length_3963_COv_1233.750977

ALIGNMENTS

>lc1|29253 NODE_1_length_3963_cov_1233.750977

Length $=3983$

Score $=4911$ bits $(5446), \quad$ Expect $=0.0$

Identities $=2723 / 2723(1008)$, Gaps $=0 / 2723(08)$

Strand=Plus / Plus 


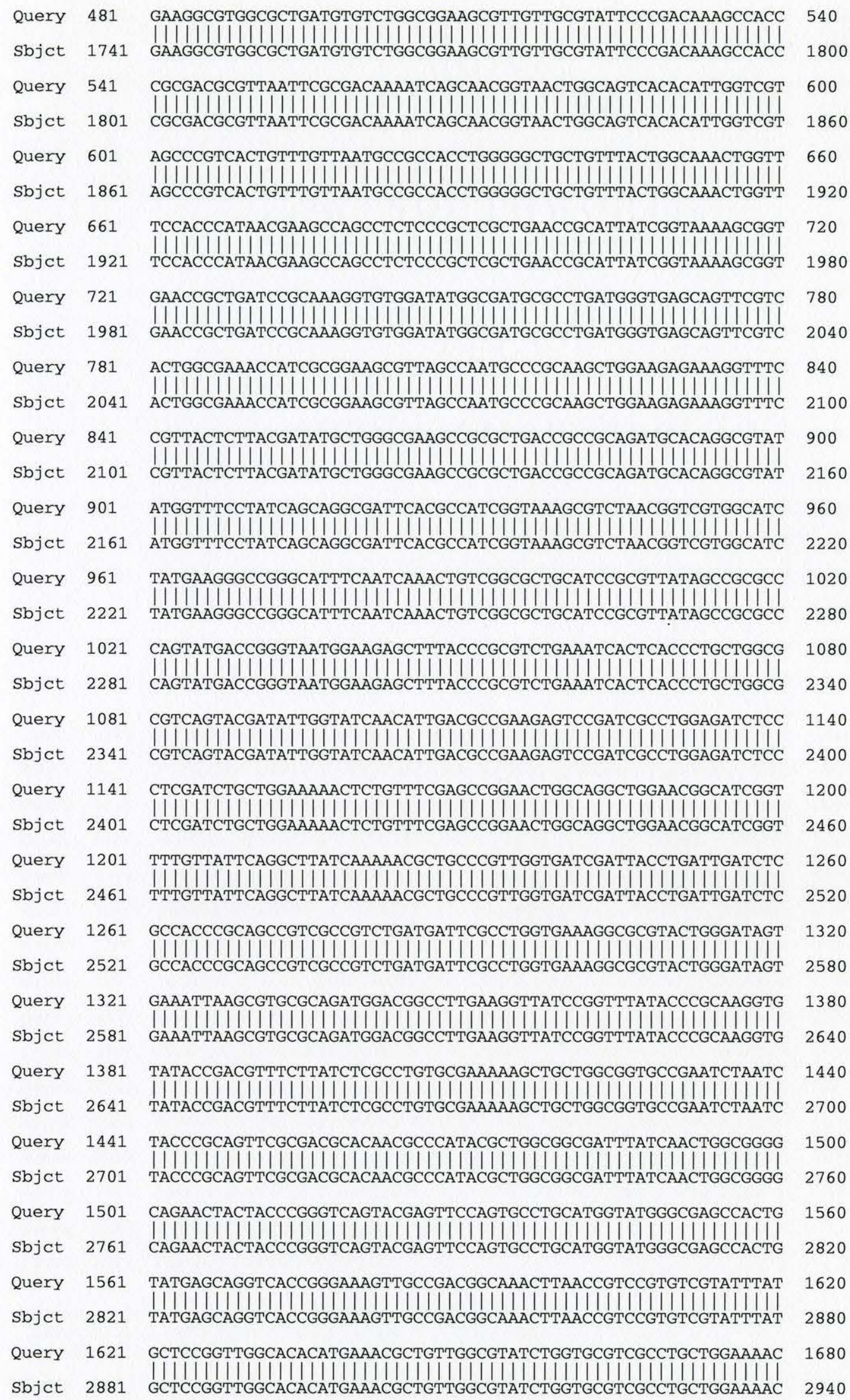




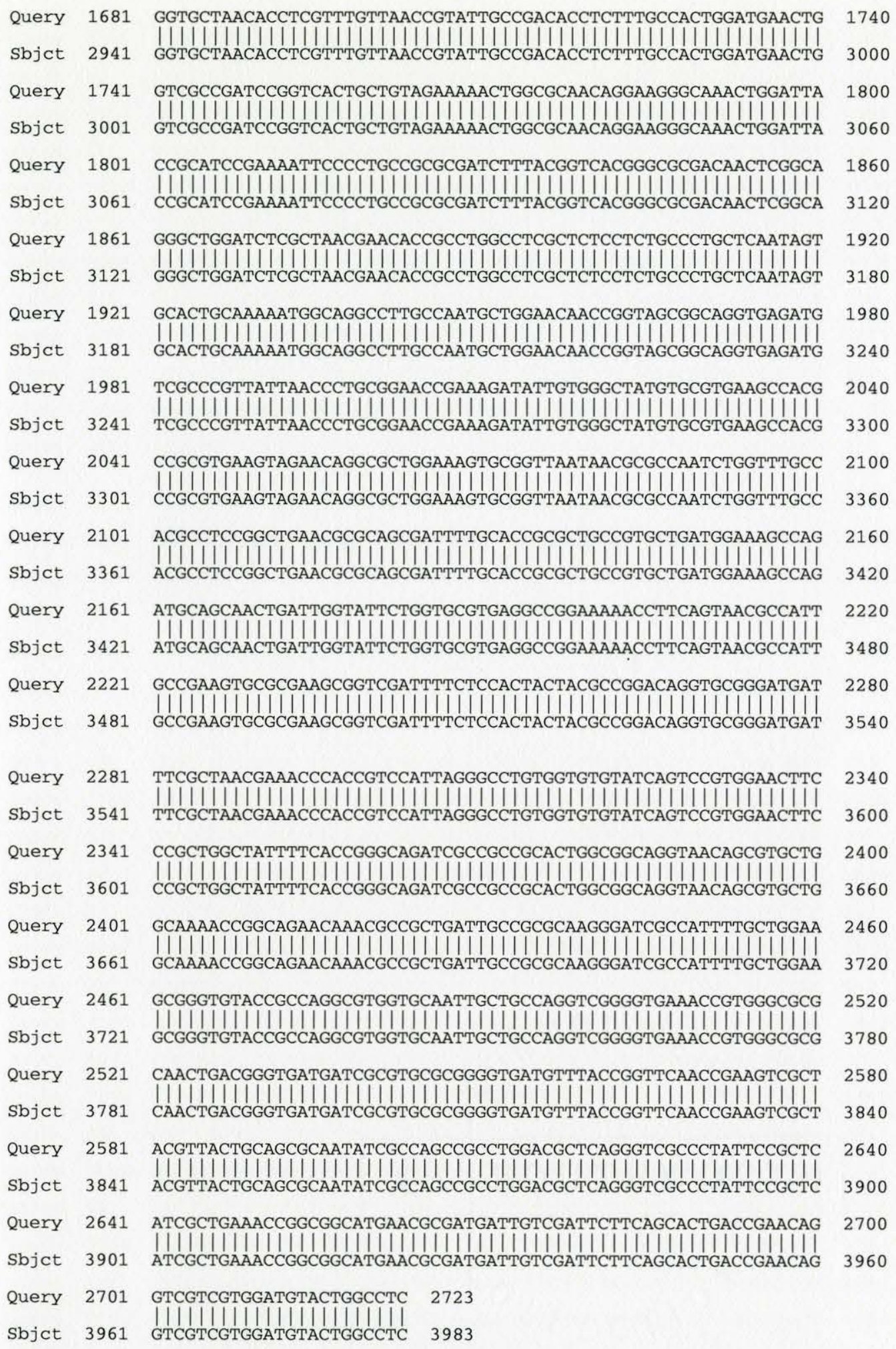

Score $=2273$ bits $(2520)$, Expect $=0.0$

Identities $=1260 / 1260(100 \%)$, Gaps $=0 / 1260(0 \%)$

Strand $=$ Plus / Plus

Query 2704 GTCGTGGATGTACTGGCCTCGGCGTTCGACAGTGCGGGTCAGCGTTGTTCGGCGCTGCGC 







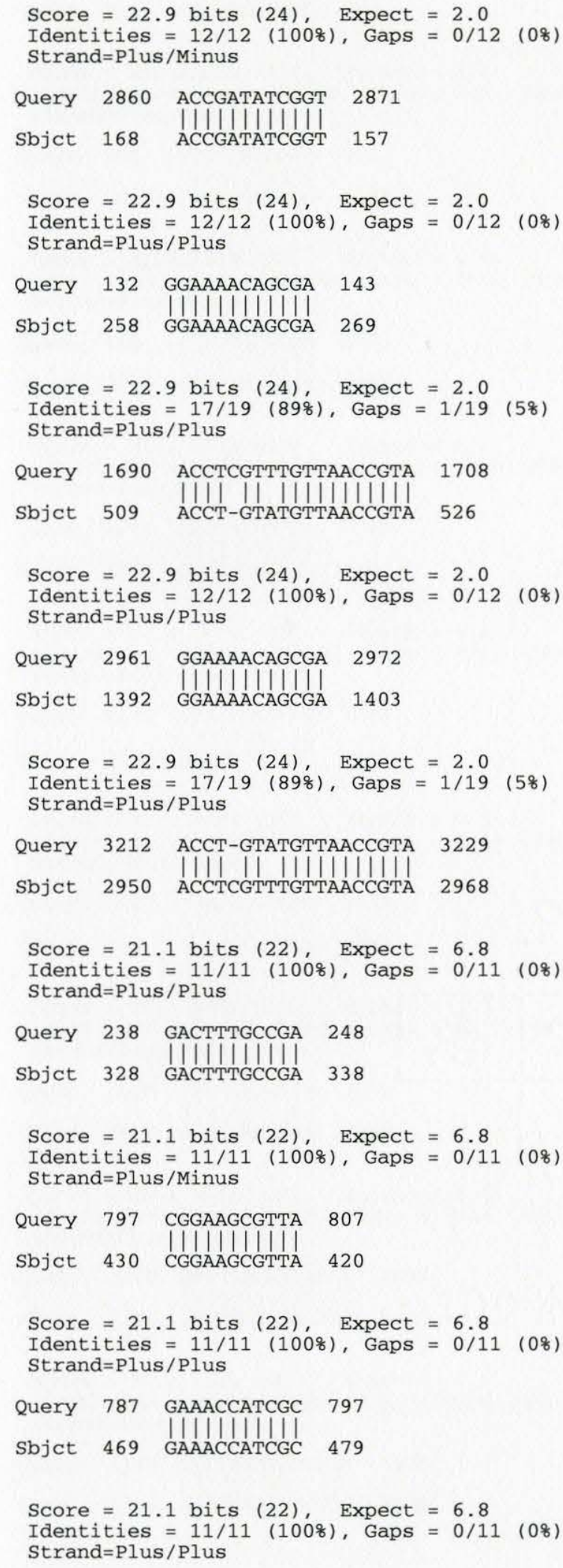




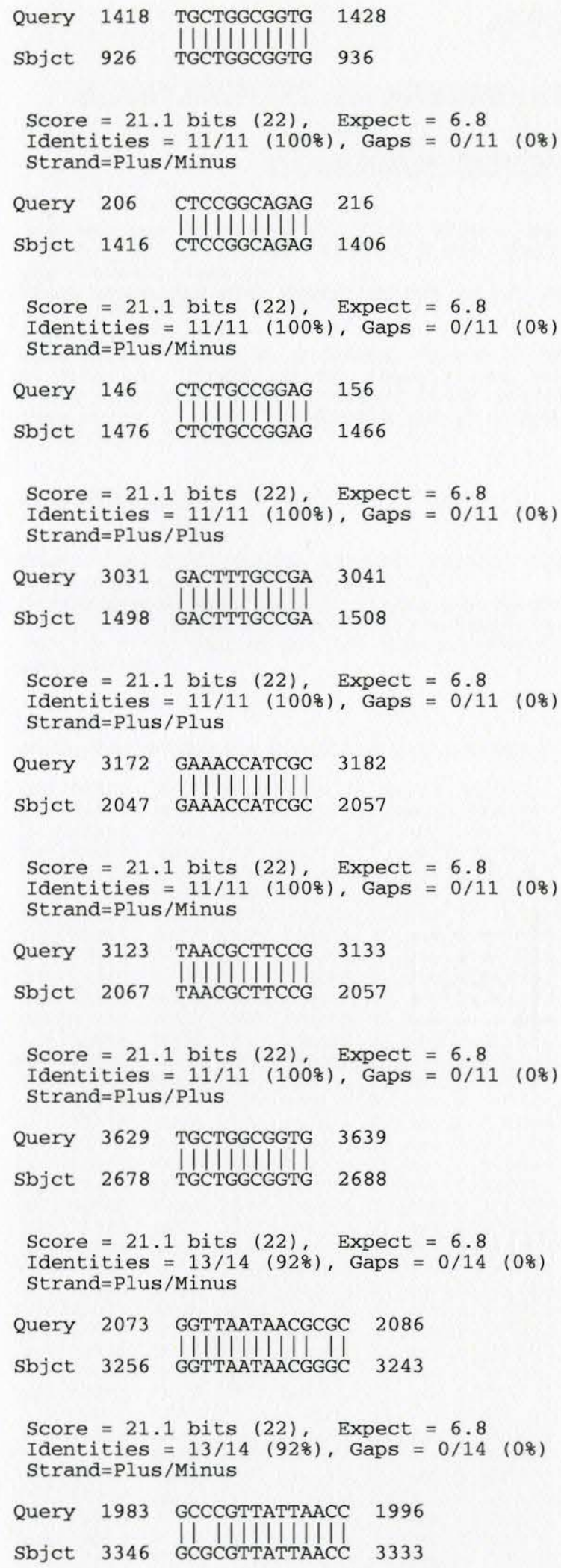




\title{
APPENDIX C
}

\section{BLAST REPORT 454 ASSEMBLED $\mathrm{k}=21$ EXPECTED COVERAGE $=4$}

\section{COVERAGE CUTOFF $=2$ VS. REFERENCE}

\begin{abstract}
velveth out_NP_415534-454_21 21 -fasta -shortPaired NP_415534-454.fasta
velvetg out_NP_415534-454_21_2_4_dir-exp_cov 4 -cov_cutoff 2 -read_trkg yes -amos_file yes -unused_reads yes

Final graph has 4253 nodes and n50 of 16, max 36, total 29411, using $1394 / 5000$ reads
\end{abstract}

BLASTN 2.2.23+

Reference: Stephen F. Altschul, Thomas L. Madden, Alejandro

A. Schaffer, Jinghui Zhang, Zheng Zhang, Webb Miller, and

David J. Lipman (1997), "Gapped BLAST and PSI-BLAST: a new

generation of protein database search programs", Nucleic

Acids Res. 25:3389-3402.

\section{RID: U30ZCU4Y114}

Query $=$ eco:b1014 putA, ECK1005, JW0999, poaA, putC; fused DNA-binding transcriptional regulator/proline dehydrogenase/pyrroline-5-carboxylate dehydrogenase (EC:1.5.99.8

1.5.1.12); K00294 1-pyrroline-5-carboxylate dehydrogenase [EC:1.5.1.12] K00318 proline dehydrogenase [EC:1.5.99.8] (N) Length $=3963$

Sequences producing significant alignments:

\begin{tabular}{|c|c|c|}
\hline & & \\
\hline & & $15 \overline{9} \_1$ \\
\hline & 976 & NODE_273_length_21_Cov_3.952381 \\
\hline & & NODE_893_1E \\
\hline & & NODE_104 $\overline{6}$ _length_2 $\overline{1} \_c o v \_6$. \\
\hline & & NODE_1086_length_23_cov_93 \\
\hline & & NODE_1139_length_21_COv_2 \\
\hline & & NODE_1229_length_21_COV_3. \\
\hline & & NODE_1278_length_21_Cov_s \\
\hline & & NODE_1407_length_21_Cov_4 \\
\hline & & NODE_1540_length_36_cov_27 \\
\hline & & NODE_1819_len \\
\hline & & NODE_1834_length_21_Cov_5. \\
\hline & & NODE_1931_length_21_Cov_4 \\
\hline & & NODE_1961_ler \\
\hline & & NODE_1994_length_21_COv_5. \\
\hline & & NODE_2005_length_21_cov_4 \\
\hline & & NODE_2082_length_21_Cov_3 \\
\hline & & NODE_2191_length_21_Cov_ \\
\hline & & NODE_2199_le \\
\hline & & NODE_2212_length_21_COV_4 \\
\hline & & NODE_2217_length_21_COv_5 \\
\hline & & NODE_2236_len \\
\hline & & NODE_2238_ler \\
\hline & & NODE_2257_len \\
\hline & & 8_length_21_cov_4 \\
\hline & & Ch_21_cov \\
\hline & & NODE_2294_length_21_Cov_3. \\
\hline & & 6_length_21_cov_4 \\
\hline & & Ch_21_cov_2 \\
\hline & & th_21_cov_4 \\
\hline & & Ch_21_cov_4 \\
\hline & & h_21_cov_7. \\
\hline & & NODE_2369_length_21_Cov_3.42 \\
\hline & & 0 length_21_cov_7. \\
\hline & & NODE_2371_1en \\
\hline & & NODE_2377_length_21_Cov_2.19 \\
\hline & & NODE_2396_length_21_COv_2 \\
\hline & & NODE_2409_length_21_COv_5. \\
\hline & & NODE_2417_length_21_COv_2 \\
\hline & & NODE_2418_length_21_Cov_6.1 \\
\hline & & NODE_2453_length_21_cov_6.571 \\
\hline & & $\mathrm{COV}_{-}$ \\
\hline
\end{tabular}

Score E

(Bits) Value

$\begin{array}{ll}66.2 & 1 \mathrm{e}-15 \\ 59.0 & 2 \mathrm{e}-13 \\ 66.2 & 1 \mathrm{e}-15 \\ 66.2 & 1 \mathrm{e}-15 \\ 66.2 & 1 \mathrm{e}-15 \\ 53.6 & 9 \mathrm{e}-12 \\ 46.4 & 1 \mathrm{e}-09 \\ 66.2 & 1 \mathrm{e}-15 \\ 66.2 & 1 \mathrm{e}-15 \\ 66.2 & 1 \mathrm{e}-15 \\ 42.8 & 2 \mathrm{e}-08 \\ 59.0 & 2 \mathrm{e}-13 \\ 66.2 & 1 \mathrm{e}-15 \\ 66.2 & 1 \mathrm{e}-15 \\ 66.2 & 1 \mathrm{e}-15 \\ 66.2 & 1 \mathrm{e}-15 \\ 66.2 & 1 \mathrm{e}-15 \\ 66.2 & 1 \mathrm{e}-15 \\ 66.2 & 1 \mathrm{e}-15 \\ 66.2 & 1 \mathrm{e}-15 \\ 66.2 & 1 \mathrm{e}-15 \\ 66.2 & 1 \mathrm{e}-15 \\ 66.2 & 1 \mathrm{e}-15 \\ 66.2 & 1 \mathrm{e}-15 \\ 66.2 & 1 \mathrm{e}-15 \\ 66.2 & 1 \mathrm{e}-15 \\ 66.2 & 1 \mathrm{e}-15 \\ 66.2 & 1 \mathrm{e}-15 \\ 66.2 & 1 \mathrm{e}-15 \\ 66.2 & 1 \mathrm{e}-15 \\ 66.2 & 1 \mathrm{e}-15 \\ 66.2 & 1 \mathrm{e}-15 \\ 66.2 & 1 \mathrm{e}-15 \\ 66.2 & 1 \mathrm{e}-15 \\ 66.2 & 1 \mathrm{e}-15 \\ 66.2 & 1 \mathrm{e}-15 \\ 66.2 & 1 \mathrm{e}-15 \\ 66.2 & 1 \mathrm{e}-15 \\ 66.2 & 1 \mathrm{e}-15 \\ 66.2 & 1 \mathrm{e}-15 \\ 66.2 & 1 \mathrm{e}-15 \\ 66.2 & 1 \mathrm{e}-15 \\ 66.2 & 1 \mathrm{e}-15 \\ 6 & \end{array}$


1cl|53017 NODE_2544_length_21_COv_3.000000

1cl 53018 NODE_2551_length_21_Cov_4.333333

$1 \mathrm{cl} 53019$ NODE_2559_length_21_cov_5.190476

1c1 53020 NODE_2566_length_21_cov_6.000000

1cl 53021 NODE_2633_length_21_Cov_3.571429

lc1 53022 NODE_2655_length_21_Cov_2.666667

1Cl 53023 NODE_2660_length_21_COv_6.238095

lc1 53024 NODE_2695_length_21_cov_3.619048

1Cl 53025 NODE_2717_length_21_Cov_3.238095

1C1 53026 NODE_2718_length_21_Cov_2.857143

lcl 53027 NODE_2773 length 21 Cov 8.380953

lc1 53028 NODE_2791_length_21_Cov_3.523809

1Cl 53029 NODE_2808_length_21_COV_4.047619

lcl 53030 NODE_2822_length_21_cov_8.142858

lc1 53031 NODE_2832_length_21_Cov_8.380953

lc1 53032 NODE_2898_length_21_COV_3.190476

lcl 53033 NODE_2899_length_21_Cov_2.428571

1cl 53034 NODE_2906_length_21_Cov_4.714286

lcl 53035 NODE_2922_length_21_Cov_4.476191

1c1 53036 NODE_2940_length_21_Cov_3.619048

1c1 53037 NODE_2949_length_21_Cov_8.047619

1cl 53038 NODE_2981_length_21_Cov_3.428571

lc1 53039 NODE_3005_length_21_cov_2.428571

lc1 53040 NODE_3009_length_21_Cov_4.476191

1cl 53041 NODE_3023_length_21_COV_4.142857

lcl 53042 NODE_3058_length_21_cov_5.952381

1c1 53043 NODE_3067_length_21_cov_3.809524

lcl 53044 NODE_3101_length_21_cov_5.047619

lcl 53045 NODE_3119_length_21_Cov_6.142857

lcl 53046 NODE_3122_length_36_Cov_2.083333

lcl 53047 NODE_3153 length 21 cov 6.190476

1c1 53048 NODE_3162_length_21_Cov_3.285714

lc1 53049 NODE_3168_length_21_Cov_4.285714

lcl 53050 NODE 3170 length_21 Cov_2.380952

1cl 53051 NODE_3181_length_21_Cov_2.619048

lc1 53052 NODE_3182_length_21_Cov_3.952381

lcl 53053 NODE_3234_length_21_cov_2.476191

lcl 53054 NODE_3245_length_21_Cov_5.238095

lc1 53055 NODE_3262_length_21_COV_4.714286

lcl 53056 NODE_3264_length_21_Cov_6.428571

1Cl 53057 NODE_3273_length_21_COv_3.190476

lc1 53058 NODE_3276_length_21_COv_5.428571

lcl 53059 NODE_3279_length_21_cov_3.571429

lc1 53060 NODE_3294_length_21_COv_4.761905

lcl 53061 NODE_3301_length_21_Cov_3.619048

1c1 53062 NODE_3302_length_21_Cov_3.761905

lc1 53063 NODE_3312_length_21_COV_4.000000

1Cl 53064 NODE_3314_length_21_cov_2.000000

1Cl 53065 NODE_3317_length_21_Cov_2.809524

lc1 53066 NODE_3330_length_21_Cov_3.238095

lcl 53067 NODE_3347_length_21_Cov_3.333333

1cl 53068 NODE_3370_length_21_COv_3.142857

lc1 53069 NODE_3387_length_21_Cov_2.714286

lc1 53070 NODE_3404_length_21_Cov_5.476191

1c1 53071 NODE_3428_length_21_Cov_5.523809

lc1 53072 NODE_3470 length_21_cov_2.571429

1C1 53073 NODE_3488_length_21_COV_2.904762

$\begin{array}{ll}66.2 & 1 \mathrm{e}-15 \\ 66.2 & 1 \mathrm{e}-15 \\ 66.2 & 1 \mathrm{e}-15 \\ 66.2 & 1 \mathrm{e}-15 \\ 66.2 & 1 \mathrm{e}-15 \\ 59.0 & 2 \mathrm{e}-13 \\ 66.2 & 1 \mathrm{e}-15 \\ 66.2 & 1 \mathrm{e}-15 \\ 66.2 & 1 \mathrm{e}-15 \\ 66.2 & 1 \mathrm{e}-15 \\ 66.2 & 1 \mathrm{e}-15 \\ 66.2 & 1 \mathrm{e}-15 \\ 66.2 & 1 \mathrm{e}-15 \\ 66.2 & 1 \mathrm{e}-15 \\ 66.2 & 1 \mathrm{e}-15 \\ 66.2 & 1 \mathrm{e}-15 \\ 59.0 & 2 \mathrm{e}-13 \\ 66.2 & 1 \mathrm{e}-15 \\ 66.2 & 1 \mathrm{e}-15 \\ 66.2 & 1 \mathrm{e}-15 \\ 66.2 & 1 \mathrm{e}-15 \\ 66.2 & 1 \mathrm{e}-15 \\ 66.2 & 1 \mathrm{e}-15 \\ 66.2 & 1 \mathrm{e}-15 \\ 66.2 & 1 \mathrm{e}-15 \\ 66.2 & 1 \mathrm{e}-15 \\ 66.2 & 1 \mathrm{e}-15 \\ 66.2 & 1 \mathrm{e}-15 \\ 66.2 & 1 \mathrm{e}-15 \\ 77.0 & 1 \mathrm{e}-18 \\ 66.2 & 1 \mathrm{e}-15 \\ 59.0 & 2 \mathrm{e}-13 \\ 59.0 & 2 \mathrm{e}-13 \\ 66.2 & 1 \mathrm{e}-15 \\ 66.2 & 1 \mathrm{e}-15 \\ 66.2 & 1 \mathrm{e}-15 \\ 66.2 & 1 \mathrm{e}-15 \\ 66.2 & 1 \mathrm{e}-15 \\ 66.2 & 1 \mathrm{e}-15 \\ 66.2 & 1 \mathrm{e}-15 \\ 66.2 & 1 \mathrm{e}-15 \\ 66.2 & 1 \mathrm{e}-15 \\ 66.2 & 1 \mathrm{e}-15 \\ 66.2 & 1 \mathrm{e}-15 \\ 66.2 & 1 \mathrm{e}-15 \\ 66.2 & 1 \mathrm{e}-15 \\ 66.2 & 1 \mathrm{e}-15 \\ 66.2 & 1 \mathrm{e}-15 \\ 66.2 & 1 \mathrm{e}-15 \\ 66.2 & 1 \mathrm{e}-15 \\ 66.2 & 1 \mathrm{e}-15 \\ 66.2 & 1 \mathrm{e}-15 \\ 66.2 & 1 \mathrm{e}-15 \\ 66.2 & 1 \mathrm{e}-15 \\ 66.2 & 1 \mathrm{e}-15 \\ 59.0 & 2 \mathrm{e}-13 \\ 66.2 & 1 \mathrm{e}-15 \\ 6 & \\ 66\end{array}$

ALIGNMENTS

>1c1|52974 NODE_11_length_21_cov_7.142857

Length $=41$

Score $=66.2$ bits $(72)$, Expect $=1 \mathrm{e}-15$

Identities $=40 / 41(97 \%)$, Gaps $=1 / 41(2 \%)$

Strand $=$ Plus / Plus

Query 1925 TGCAAAAATGGCAGGCCTTG-CCAATGCTGGAACAACCGGT

1964

Sbjet 1

TGCAAAAATGGCAGGCCTTGTCCAATGCTGGAACAACCGGT

41

Score $=21.1$ bits (22), Expect $=0.052$

Identities $=11 / 11(100 \%)$, Gaps $=0 / 11(0 \%)$

Strand $=$ Plus / Plus

Query 329 TGCTGGAACAA 339

sbjet 26 TGCTGGAACAA 36 
Length $=41$

Score $=59.0$ bits $(64)$, Expect $=2 e-13$

Identities $=39 / 41(95 \%)$, Gaps $=2 / 41(4 \%)$

Strand=Plus/Plus

Query 3837 TGTTTCGGTGCAGGGTTTTG-CCCGT-GGCGAAAGCAATAT 3875

Sbjet 1 TGTTTCGGTGCAGGGTTTTGACCCGTCGGCGAAAGCAATAT 41

>1c1|52976 NODE_273_length_21_COv_3.952381

Length $=41$

Score $=66.2$ bits $(72), \quad$ Expect $=1 \mathrm{e}-15$

Identities $=40 / 41(97 \%)$, Gaps $=1 / 41(2 \%)$

Strand=Plus /Minus

Query 2040 GCCGCGTGAAGTAGAACAGG-CGCTGGAAAGTGCGGTTAAT 2079

Sbjet 41 GCCGCGTGAAGTAGAACAGGACGCTGGAAAGTGCGGTTAAT 1

>1cl|52977 NODE_893_length_21_Cov_5.047619

Length $=41$

Score $=66.2$ bits $(72)$, Expect $=1 \mathrm{e}-15$

Identities $=40 / 41(97 \%)$, Gaps $=1 / 41(2 \%)$

Strand $=$ Plus $/$ Minus

Query 3196 TCGGCCCATGTTGGTAACCT-GTATGTTAACCGTAATATGG

sbjet 41 TCGGCCCATGTTGGTAACCTAGTATGTTAACCGTAATATGG

1

Score $=26.5$ bits $(28)$, Expect $=0.001$

Identities $=17 / 19(898)$, Gaps $=0 / 19(0 \%)$

Strand=Plus $/$ Minus

$\begin{array}{llll}\text { Query } & 1690 & \text { ACCTCGTTTGTTAACCGTA } & 1708 \\ \text { Sbjet } & 25 & |\|||||||||||||||| & \\ \text { ACCTAGTATGTTAACCGTA } & 7\end{array}$

$>1$ l| 52978 NODE_1046_length_21_cov_6.666667

Length $=41$

Score $=66.2$ bits $(72)$, Expect $=1 \mathrm{e}-15$

Identities $=40 / 41(978)$, Gaps $=1 / 41(2 \%)$

Strand=Plus/Minus

Query 3185 AGGTCACTGGCTCGGCCCAT-GTTGGTAACCTGTATGTTAA

3224

sbjet 41 AGGTCACTGGCTCGGCCCATAGTTGGTAACCTGTATGTTAA

1

>1c1|52979 NODE_1086_length_23_Cov_93.173912

Length $=43$

Score $=53.6$ bits $(58)$, Expect $=9 \mathrm{e}-12$

Identities $=40 / 43(93 \%)$, Gaps $=3 / 43(68)$

Strand=Plus/Minus

Query 2583 GTTACTGCAGCGCAA-TATCG-CCAGCCGCCTGGACGCTCAGG

Sbjet 42

$>1 \mathrm{cl} \mid 52980$ NODE_1139_length_21_Cov_27.714285

Length $=41$

Score $=46.4$ bits $(50)$, Expect $=1 \mathrm{e}-09$

Identities $=29 / 30(968)$, Gaps $=1 / 30(38)$

Strand=Plus $/$ Minus

Query 1 ATGGGAACC-ACCACCATGGGGGTTAAGCT

Sbjet 30 ATGGGAACCTACCACCATGGGGGTAAAGCT 


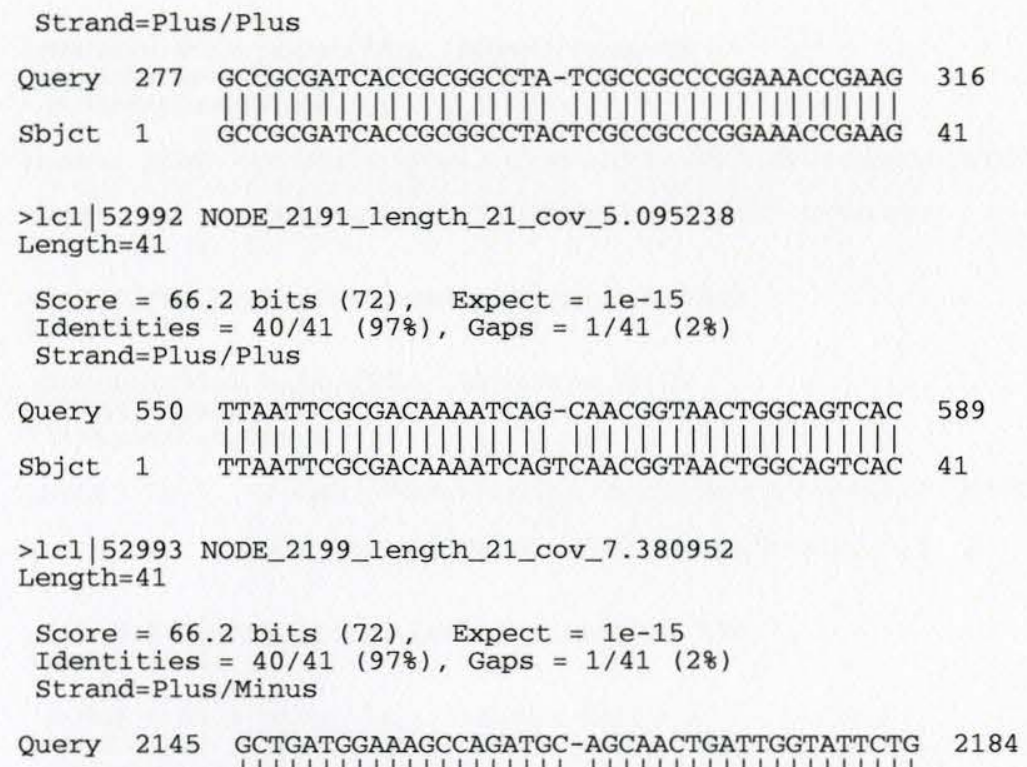


$>1 \mathrm{cl} \mid 53005$ NODE_2351_length_21_Cov_4.761905

Length $=41$

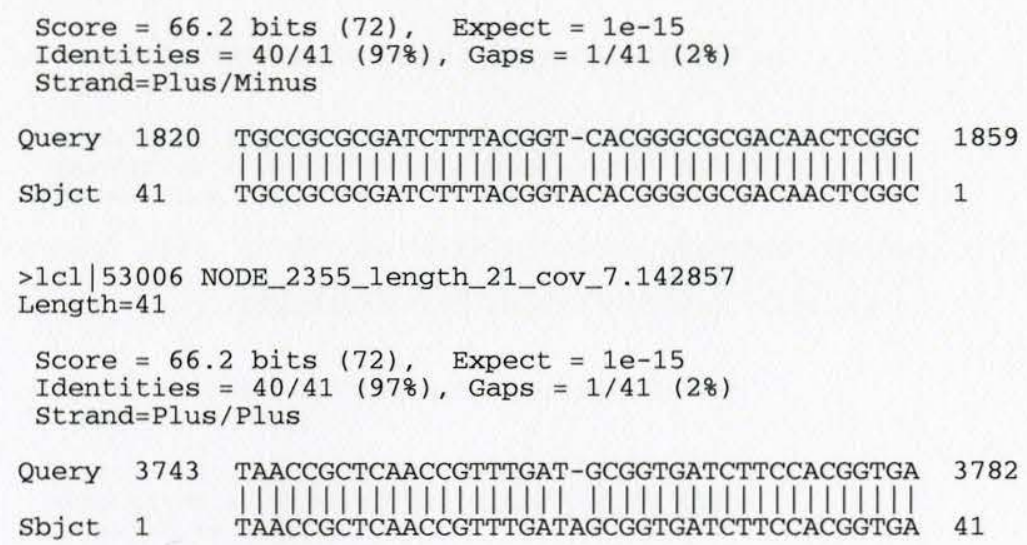

>1c1|53008 NODE_2370_length_21_Cov_7.190476 Length $=41$

Score $=66.2$ bits $(72)$, Expect $=1 \mathrm{e}-15$

Identities $=40 / 41(97 \%)$, Gaps $=1 / 41(2 \%)$

Strand=Plus/Plus

Query 3319 TACCGTCTGCTGGCGAATCG-CCCGGAAAGTGCGCTGGCAG 3358 Sbjet 1

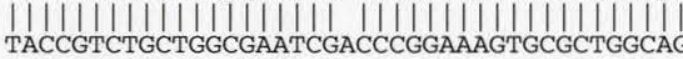
41

>1c1|53009 NODE_2371_length_21_Cov_4.190476 Length $=41$

Score $=66.2$ bits $(72), \quad$ Expect $=1 \mathrm{e}-15$

Identities $=40 / 41(97 \%)$, Gaps $=1 / 41(2 \%)$

Strand $=$ Plus $/$ Plus

Query 3476 TATGTACGCAATATGGCGAG-CTGGCGCAGGCAGGAACACA 3515 Sbjet 1 TATGTACGCAATATGGCGAGTCTGGCGCAGGCAGGAACACA

$>1$ l| 53010 NODE_2377_length_21_Cov_2.190476 Length $=41$

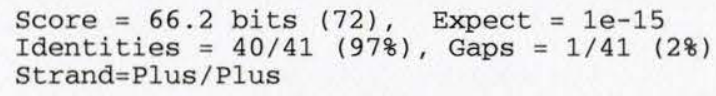


Query 2998 ACCTTTGTCGCCCCGACGCT-GATCGAACTGGATGACTTTG 3037

sbjet 41 ACCTTTGTCGCCCCGACGCTCGATCGAACTGGATGACTTTG

>1c1|53012 NODE_2409_length_21_Cov_5.142857

Length $=41$

Score $=66.2$ bits $(72)$, Expect $=1 \mathrm{e}-15$

Identities $=40 / 41(978)$, Gaps $=1 / 41(28)$

Strand $=$ Plus / Plus

Query 3081 GCGTTACAACCGTAACCAGC-TACCAGAGCTGATCGAGCAG 3120 Sbjet 1

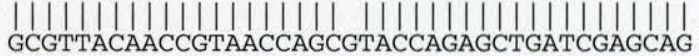
41

>1c1|53013 NODE_2417_length_21_Cov_2.238095 Length $=41$

Score $=66.2$ bits $(72)$, Expect $=1 \mathrm{e}-15$

Identities $=40 / 41(97 \%)$, Gaps $=1 / 41(2 \%)$

Strand=Plus / Plus

Query 1971 AGGTGAGATGTCGCCCGTTA-TTAACCCTGCGGAACCGAAA

2010

Sbjet 1 AGGTGAGATGTCGCCCGTTAGTTAACCCTGCGGAACCGAAA

41

>1C1|53014 NODE_2418_length_21_cov_6.190476

Leng th $=41$

Score $=66.2$ bits $(72)$, Expect $=1 \mathrm{e}-15$

Identities $=40 / 41(97 \%)$, Gaps $=1 / 41(2 \%)$

Strand=Plus/Plus

Query 3080 TGCGTTACAACCGTAACCAG-CTACCAGAGCTGATCGAGCA

3119

sbjet 1 TGCGTTACAACCGTAACCAGTCTACCAGAGCTGATCGAGCA

41

>1cl|53015 NODE_2453_length_21_cov_6.571429

Length $=41$

Score $=66.2$ bits $(72)$, Expect $=1 \mathrm{e}-15$

Identities $=40 / 41(97 \%)$, Gaps $=1 / 41$ (28)

Strand=Plus $/$ Minus

Query 764 TGGGTGAGCAGTTCGTCACT-GGCGAAACCATCGCGGAAGC

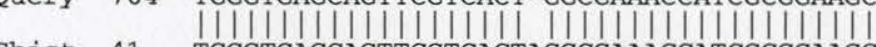

sbjet 41 TGGGTGAGCAGTTCGTCACTAGGCGAAACCATCGCGGAAGC

803 1

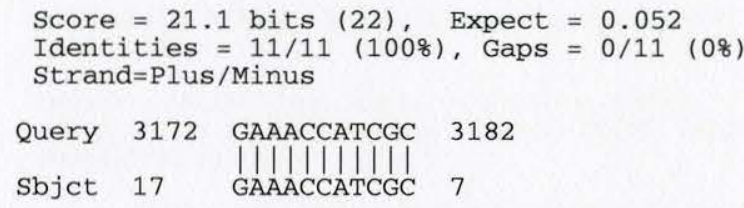


Query 2349 TATTTTCACCGGGCAGATCG-CCGCCGCACTGGCGGCAGGT

>1C1|53018 NODE_2551_length_21_Cov_4.333333

Length $=41$

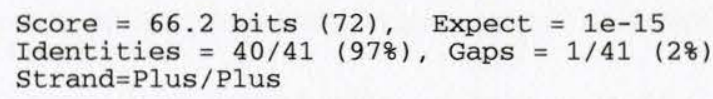

>1C1|53019 NODE_2559_length_21_COV_5.190476

Length $=41$

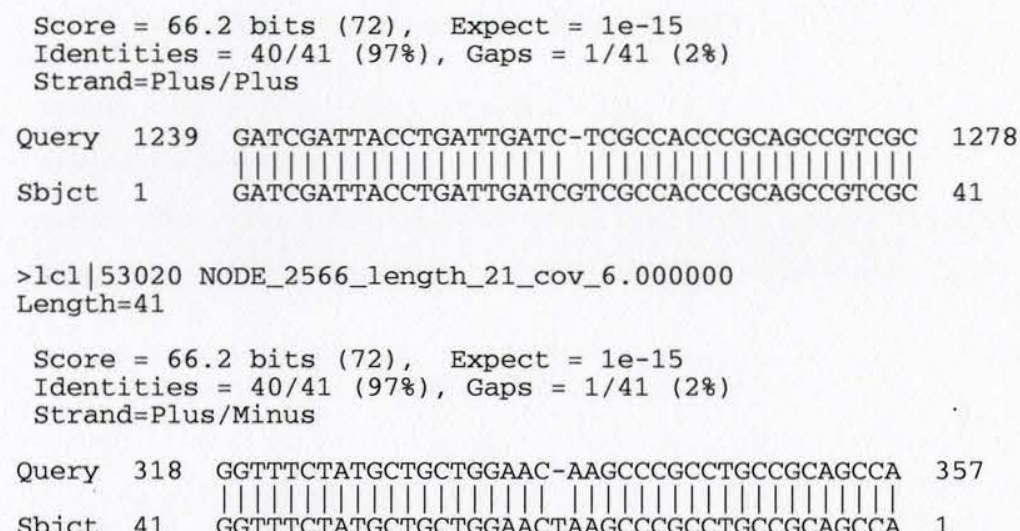

>1cl|53022 NODE_2655_length_21_Cov_2.666667

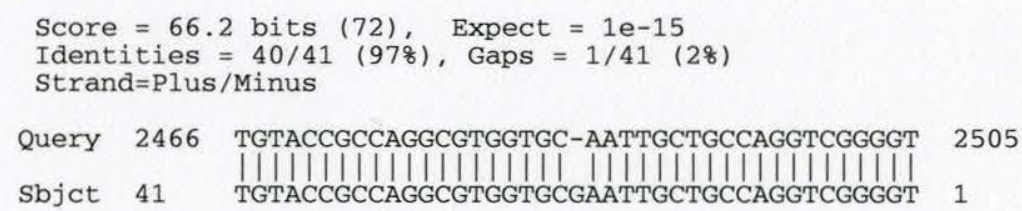


$>1 \mathrm{cl} \mid 53043$ NODE_3067_length_21_cov_3.809524

Length $=41$

Score $=66.2$ bits $(72)$, Expect $=1 \mathrm{e}-15$

Identities $=40 / 41(97 \%)$, Gaps $=1 / 41(28)$

Strand=Plus /Minus

Query 775 TTCGTCACTGGCGAAACCAT-CGCGGAAGCGTTAGCCAATG 814

Sbjet 41 TTCGTCACTGGCGAAACCATACGCGGAAGCGTTAGCCAATG 1

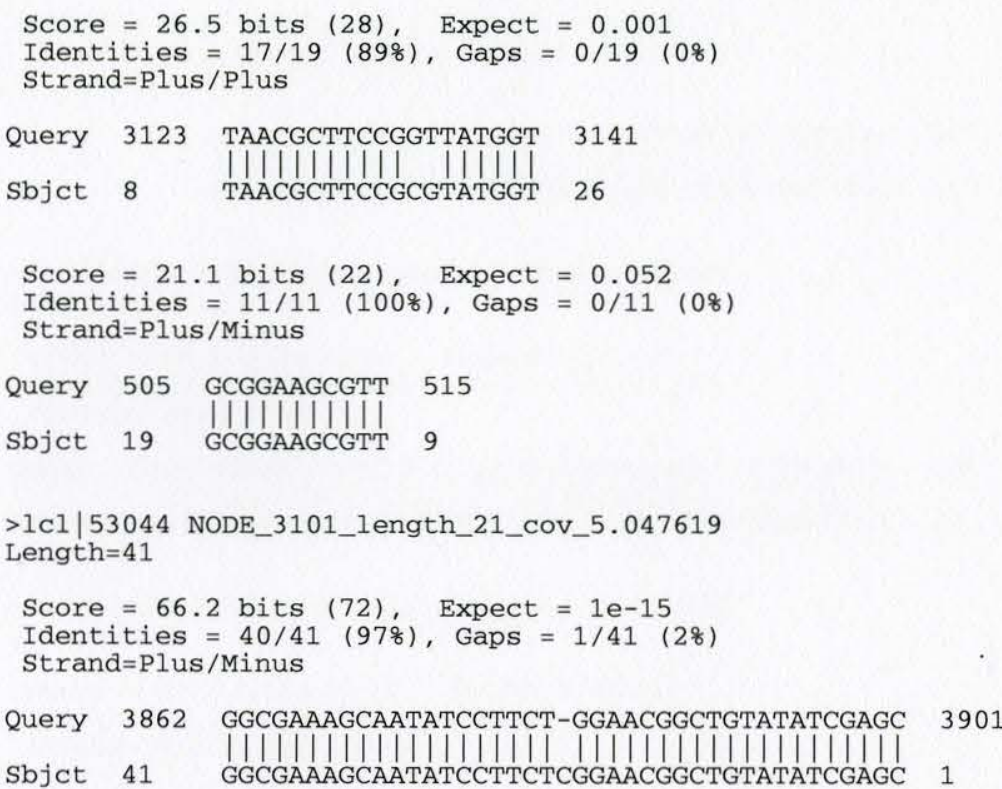

>1c1|53045 NODE_3119_length_21_cov_6.142857

Length $=41$

Score $=66.2$ bits $(72)$, Expect $=1 \mathrm{e}-15$

Identities $=40 / 41(97 \%)$, Gaps $=1 / 41(2 \%)$

Strand=Plus/Minus

Query 356 CAGTTGCTGAACAGGCGCAC-AAACTGGCGTATCAGCTGGC 395

Sbjet 41 CAGTTGCTGAACAGGCGCACGAAACTGGCGTATCAGCTGGC 1

$>1 \mathrm{cl|} 53046$ NODE_3122_length_36_cov_2.083333

Length $=56$

Score $=77.0$ bits $(84)$, Expect $=1 \mathrm{e}-18$

Identities $=53 / 56(948)$, Gaps $=3 / 56(58)$

Strand $=$ Plus / Plus

Query 966 AGGGCCGGGCATTTCAA-TC-AAACTGTCGGCGCT-GCATCCGCGTTATAGCCGCG 1018

Sbjet 1|||||||||||||||||||||||||||||||||||||||||||||||||||| $\mid$

>1c1|53047 NODE_3153_length_21_Cov_6.190476

Length $=41$

Score $=66.2$ bits $(72)$, Expect $=1 \mathrm{e}-15$

Identities $=40 / 41(97 \%)$, Gaps $=1 / 41$ (28)

Strand=Plus/Minus

Query 2352 TTTCACCGGGCAGATCGCCG-CCGCACTGGCGGCAGGTAAC

2391

sbjet 41 TTTCACCGGGCAGATCGCCGTCCGCACTGGCGGCAGGTAAC 1

>1cl|53048 NODE_3162_length_21_Cov_3.285714

Length $=41$ 


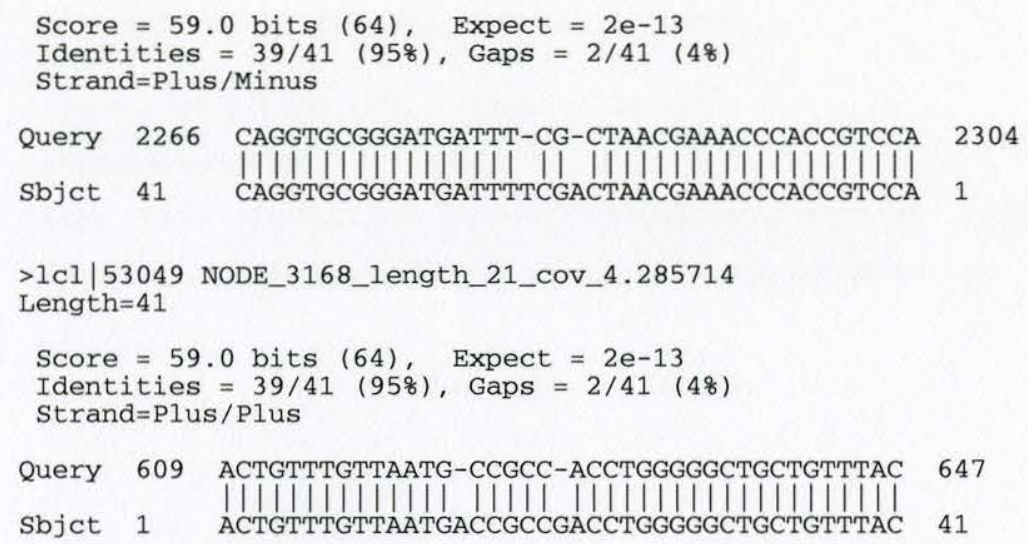


>1c1|53061 NODE_3301_length_21_cov_3.619048 Length $=41$

Score $=66.2$ bits $(72)$, Expect $=1 \mathrm{e}-15$

Identities $=40 / 41(97 \%)$, Gaps $=1 / 41(2 \%)$

Strand=Plus/Plus

Query 2659 ATGAACGCGATGATTGTCGA-TTCTTCAGCACTGACCGAAC

>1cl|53062 NODE_3302_length_21_cov_3.761905

Length $=41$

Score $=66.2$ bits $(72)$, Expect $=1 \mathrm{e}-15$

Identities $=40 / 41(978)$, Gaps $=1 / 41(28)$

Strand $=$ Plus $/$ Plus

Query 2741 GTCAGCGTTGTTCGGCGCTG-CGCGTGCTGTGCCTGCAAGA

$>1 \mathrm{cl} \mid 53063$ NODE_3312_length_21_cov_4.000000 Length $=41$

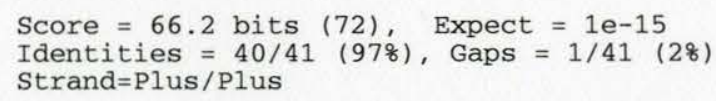

Query 632 GGGGGCTGCTGTTTACTGGC-AAACTGGTTTCCACCCATAA ||||||||||||||||||||| ||||||||||||||||||||||

>1c1|53064 NODE 3314 length_21_cov_2.000000

Length $=41$

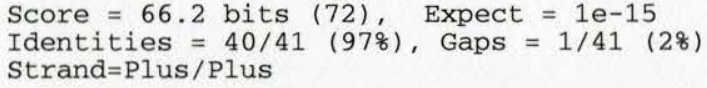

Query 3893 ATATCGAGCGTTCGCTGAGT-GTGAATACCGCTGCCGCTGG

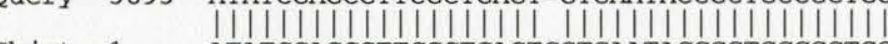

>1c1|53065 NODE_3317_1ength_21_Cov_2.809524 Length $=41$

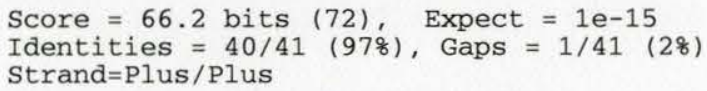

>1c1|53066 NODE_3330_length_21_Cov_3.238095 Length $=41$

Score $=66.2$ bits $(72)$, Expect $=1 \mathrm{e}-15$

Identities $=40 / 41(97 \%)$, Gaps $=1 / 41(2 \%)$

Strand $=$ Plus /Plus

Query 2277 TGATTTCGCTAACGAAACCC-ACCGTCCATTAGGGCCTGTG

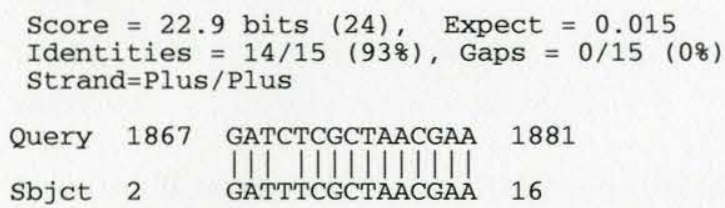


Score $=59.0$ bits $(64)$, Expect $=2 \mathrm{e}-13$

Identities $=39 / 41(95 \%)$, Gaps $=2 / 41(48)$

Strand $=\mathrm{Pl}$ us $/$ Minus

Query 3649 GTAC-TGTGGCCGGATGACG-CGCTGCATCGTCAGTTAGTG

sbjet 41 GTACGTGTGGCCGGATGACGACGCTGCATCGTCAGTTAGTG

>1c1|53073 NODE_3488_length_21_Cov_2.904762

Length $=41$

Score $=66.2$ bits $(72), \quad$ Expect $=1 \mathrm{e}-15$

Identities $=40 / 41(97 \%)$, Gaps $=1 / 41(28)$

Strand $=\mathrm{Pl}$ us $/ \mathrm{Pl}$ us

Query 407 GTAATCnnnnnnnTGCCAGT-GGTCGCGCAGGTATGGTCCA 446 
APPENDIX D

\title{
BLAST REPORT 454 ASSEMBLED $\mathrm{k}=31$ EXPECTED COVERAGE $=24$ \\ COVERAGE CUTOFF $=12$ VS. REFERENCE
}

\author{
velveth out_NP_415534-454_31 31 -fasta -shortPaired NP_415534-454.fasta \\ amos_file yes -unused_reads yes \\ BLASTN 2.2.23+ \\ Reference: Stephen F. Altschul, Thomas L. Madden, Alejandro \\ A. Schaffer, Jinghui Zhang, Zheng Zhang, Webb Miller, and \\ David J. Lipman (1997), "Gapped BLAST and PSI-BLAST: a new \\ generation of protein database search programs", Nucleic \\ Acids Res. 25:3389-3402.
}

velvetg out_NP_415534-454_31_12_24_dir -exp_cov 24 -cov_cutoff 12 -read_trkg yes -

Final graph has 22 nodes and $n 50$ of 267, max 461, total 3130, using 5000/5000 reads

RID: U2ZPZ325112

Query = eco:b1014 putA, ECK1005, JW0999, poaA, putC; fused DNA-binding transcriptional regulator/proline

dehydrogenase/pyrroline-5-carboxylate dehydrogenase (EC:1.5.99.8

1.5.1.12); K00294 1-pyrroline-5-carboxylate dehydrogenase

[EC:1.5.1.12] K00318 proline dehydrogenase [EC:1.5.99.8] (N)

Length $=3963$

Sequences producing significant alignments:

1c1|63088 NODE_1_length_393_cov_219.491089

lc1 63089 NODE_2_length_282_Cov_195.992905

1c1 63090 NODE_3_length_267_Cov_206.041199

lc1 63091 NODE_4_length_206_Cov_205.684464

lc1 63092 NODE_5_length_158_Cov_201.348099

lc1 63093 NODE_6_length_291_cov_199.041245

lc1 63094 NODE_7_length_461_cov_209.563995

lc1 63095 NODE_8_length_243_cov_213.312759

lc1 63096 NODE_9_length_98_Cov_212.948975

lcl 63097 NODE_10_length_148_cov_229.418915

lc1 63098 NODE_11_length_76_Cov_221.750000

Icl 63099 NODE_12_length_62_Cov_208.145157

lc1 63100 NODE_13_length_73_Cov_221.095886

lcl 63101 NODE_15_length_142_cov_198.697189

lc1 63102 NODE_16_length_65_cov_216.415390

lc1 63103 NODE_17_length_101_Cov_213.732666

ALIGNMENTS

$>1$ cl|63088 NODE_1_length_393_cov_219.491089

Length $=423$
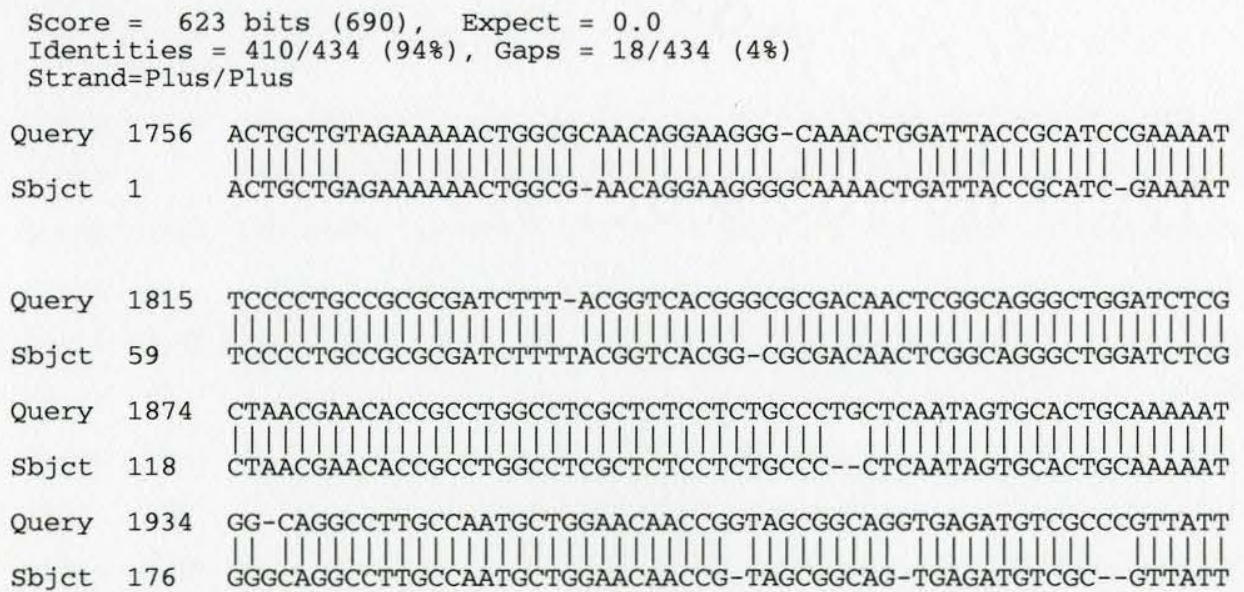


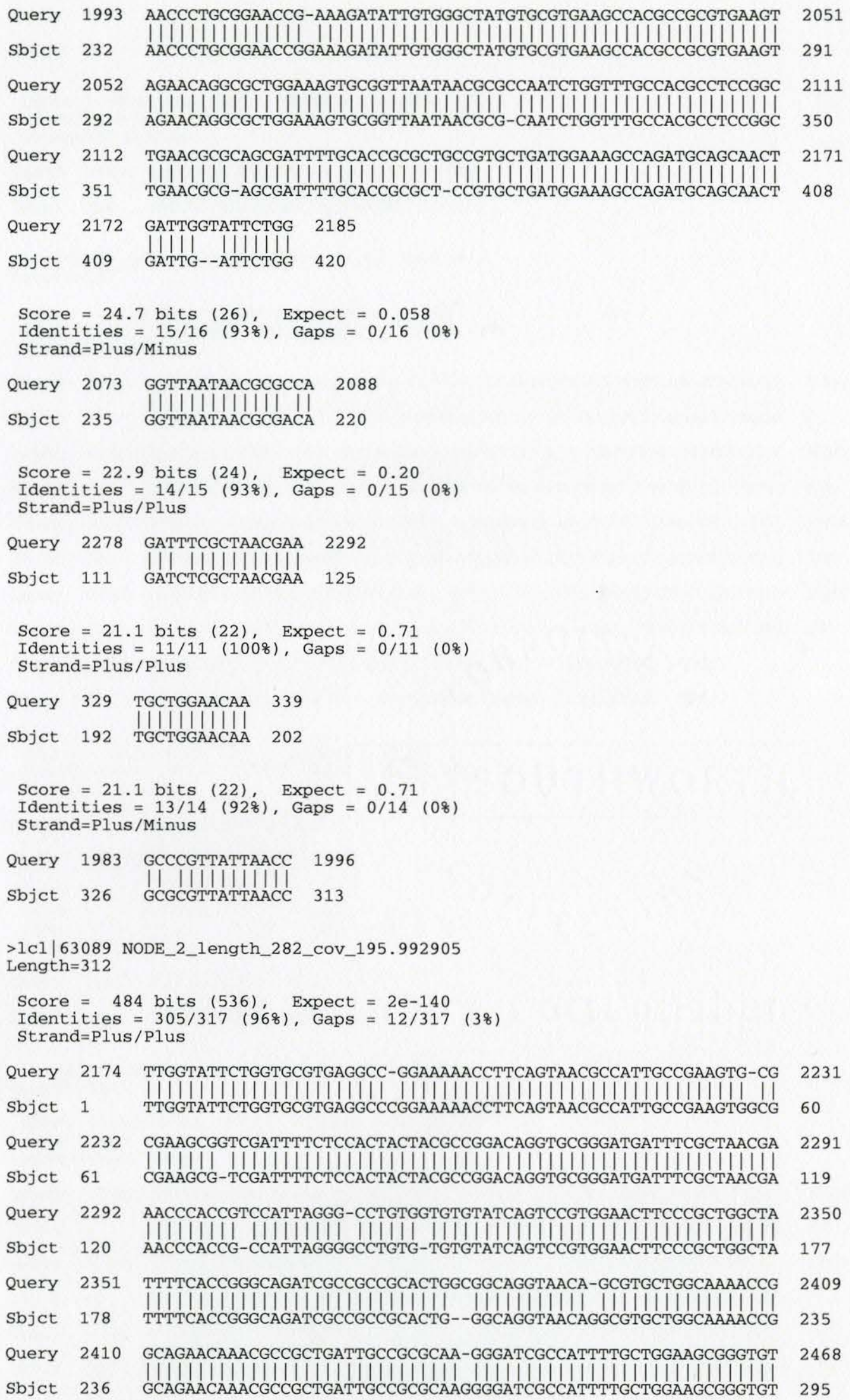




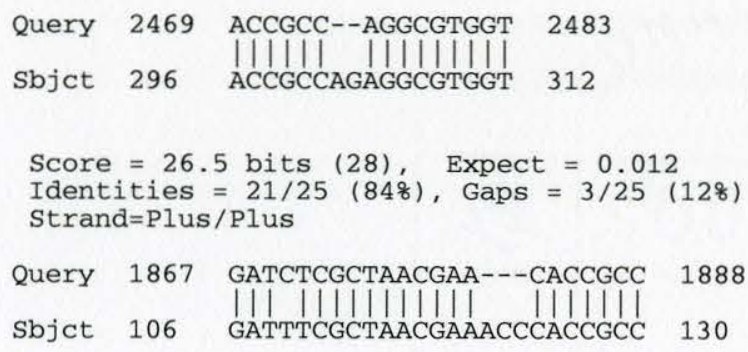




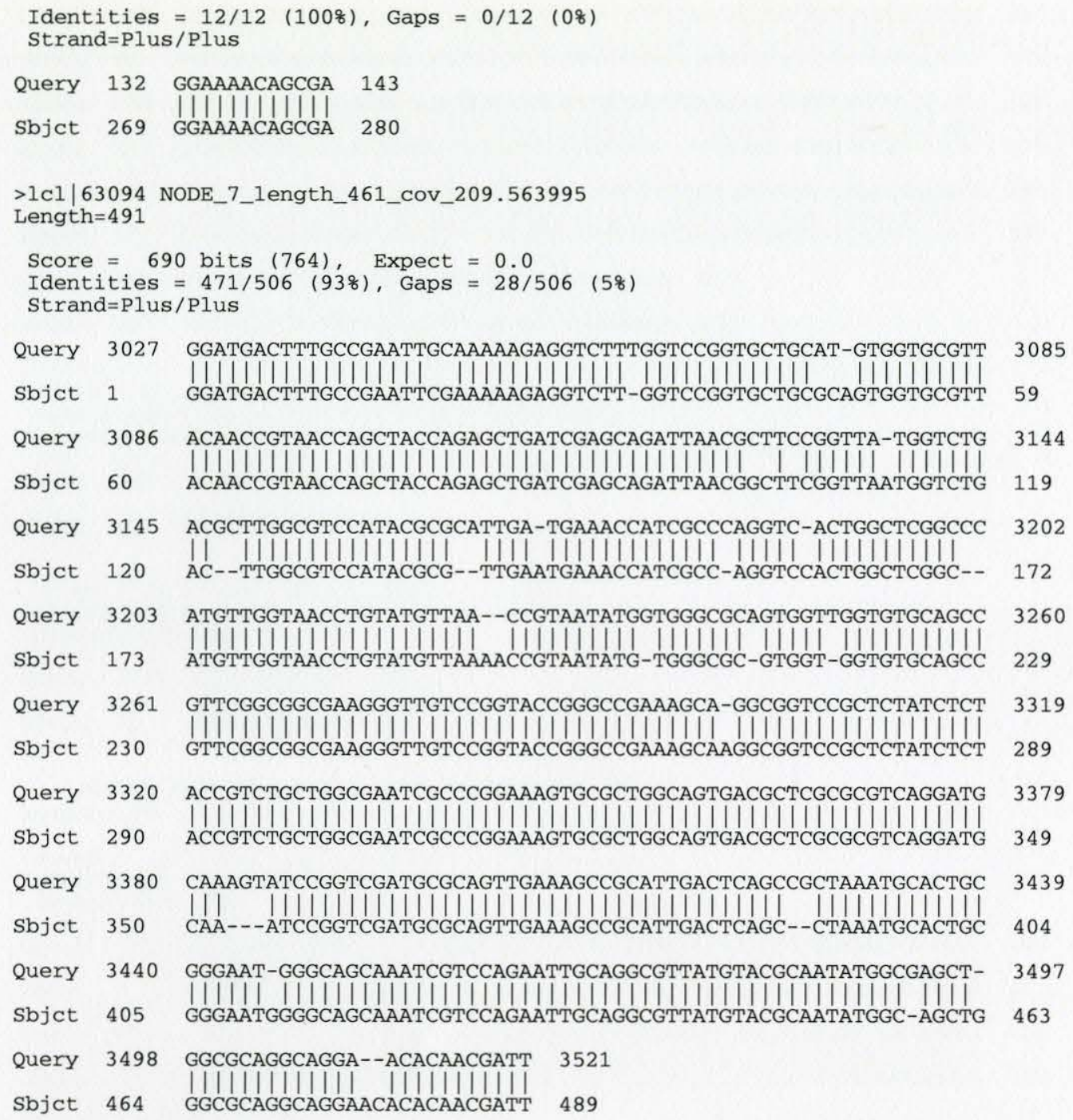




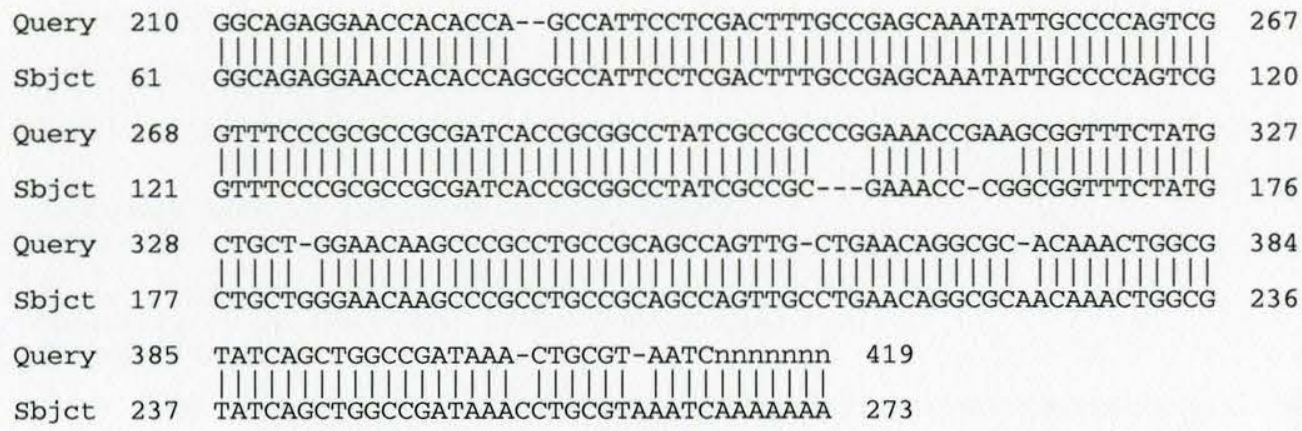

Score $=21.1$ bits (22), Expect $=0.45$

Identities $=11 / 11(100 \%)$, Gaps $=0 / 11(0 \%)$

Strand $=\mathrm{Plus} /$ Minus

Query 146 CTCTGCCGGAG 156

sbjet 67 CTCTGCCGGAG 57

Score $=21.1$ bits (22), Expect $=0.45$

Identities $=11 / 11(1008)$, Gaps $=0 / 11(08)$

Strand=Plus /Plus

Query 3031 GACTTTGCCGA 3041

Sbjet $91 \quad$ GACTTTGCCGA 101

>1c1|63096 NODE_9_length_98_Cov_212.948975

Length $=128$

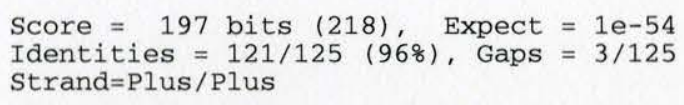

Query 462 GTTTTCGCTGTCATCGCAGGAAGGCGTGGCGCTGATGTGTCTGGC-GGAAGCGTTGTTGC

Sbjet 2 GTTTTCGCTGTCATCGCAGGAGGCGTGGAGCTGATGTGTCTGGCGGAAGCGTTGTTGC

520

Query 521 GTATTCCCGACAAAGCCA-CCCGCGACGCG-TTAATTCGCGACAAAATCAGCAACGGTAA Sbjet 62 GTATTCCCGACAAAGCCACCCCGCGACGCGTTTAATTCGCGACAAAATCAGCAACGGTAA

Query 579 CTGGC 583

Sbjet $122 \stackrel{|||| \mid}{\text { CTGGC }} 126$

>1c1|63097 NODE_10_length_148_cov_229.418915

Length $=178$

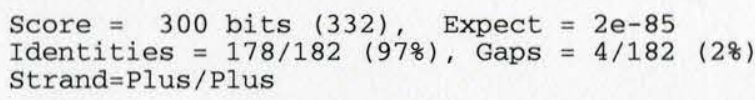

Query 831 GAAAGGTTTCCGTTACTCTTACGATATGCTGGGCGAAGCCGCGCTGACCGCCGCAGATGC

Sbjet 1

sbjet 61 ACAG-CGTATATGGTTTCCTATCAGCAGGCGATTCACGCCATCGGTAAAGCGTCTAACGG

Query 951 TCGTGGCATCTATGAAGGGCCGGGCATTTCAATCAAACTGTCGGCGCTGCATCCGCGTTA

$\begin{array}{llll}\text { Query } & 1011 & \text { TA } & 1012 \\ \text { Sbjct } & 177 & \text { TA } & 178\end{array}$


Strand $=$ Plus $/$ Plus

$\begin{array}{llll}\text { Query } & 101 & \text { AGCAGGCGATT } & 111 \\ \text { Sbjet } & 83 & \text { AGCAGGCGATT } & 93\end{array}$

>1c1|63098 NODE_11_length_76_cov_221.750000

Length $=106$

Score $=156$ bits $(172)$, Expect $=3 e-42$

Identities $=101 / 106(958)$, Gaps $=5 / 106(4 \%)$

Strand=Plus/Plus

Query 1026 TGACCGGGTAATGGAAGAGCTTTACCCGCGTCTGAAATCACTCACCCTGCTGGCGCGTCA 1085

Sbjet 4 TGACCGGGTAATGGAAGAGCTTTACCCGCGTCTGAAATCACTCACCCTGCTGGCGCGTCA

63

Query 1086 GTACGATATTGGTATCAAC-ATTGACGCCGAAGAGTCCGA-TCGCC 1129

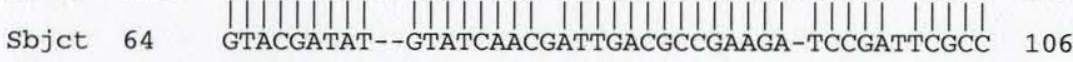

>1c1 |63099 NODE_12_length_62_Cov_208.145157

Length $=92$

Score $=129$ bits $(142)$, Expect $=4 \mathrm{e}-34$

Identities $=89 / 94(94 \%)$, Gaps $=5 / 94(58)$

Strand=Plus/Minus

Query 3611 TGACTCAG-CTCGCCGCCGTGCTGGCGGTGGGC-AGCCAGGTACTGTGGCCGGATGACGC luer

sbjet 92 TGACTCAGTCTCGCCGCCGTGCTGGCGGTGGGCGAGCAGGTACTGTGGCC-GATGACGC 34

Query 3669 G-CTGCATCGTCAGTTAGTGAAGGCATTGCCATC 3701

Sbjet 33 GCCTGCATCGTCAGTTAG-GAAGGCATTGCCATC 1

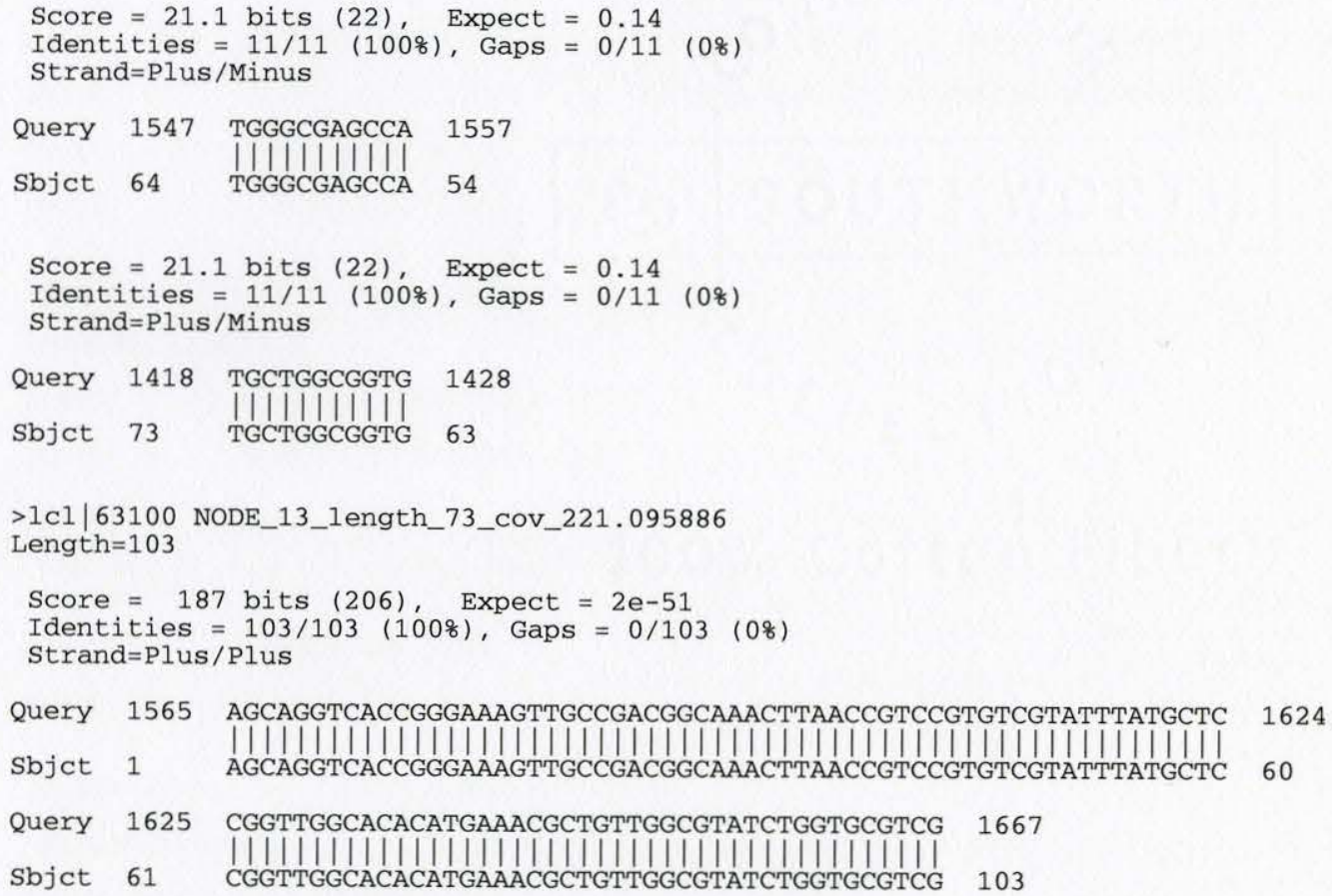




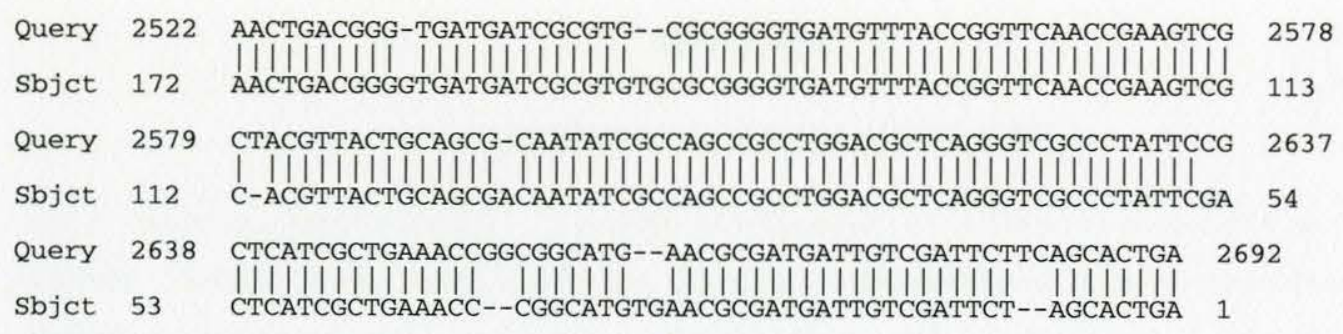

>lcl|63102 NODE_16_length_65_Cov_216.415390

Length $=95$

Score $=114$ bits $(126), \quad$ Expect $=8 \mathrm{e}-30$

Identities $=86 / 97(888)$, Gaps $=7 / 97(78)$

Strand=Plus/Plus
Query 1502 AGAACTACTACCCGGGTCAGTACG--AGTTCCAGTGCCTGCATGGTATGGGC--GAGCCA 1557




\section{APPENDIXE}

\section{METASIM “EXACT” EXCERPT}

$>r 1.1$ SOURCES $=\{\mathrm{KEY}=0 \mathrm{f} 5 \mathrm{~d} 3 \mathrm{~d} 4 \mathrm{~b} \ldots \mathrm{fw}, 2703-3330\} \mid$ ERRORS $=\{\} \mid$ SOURCE_1="eco:b1014 putA, ECK1005, JW0999, poaA, putC; fused DNA-binding transcriptional regulator/proline dehydrogenase/pyrroline-5-carboxylate dehydrogenase (EC:1.5.99.8 1.5.1.12); K00294 1pyrroline-5-carboxylate dehydrogenase [EC:1.5.1.12] K00318 proline dehydrogenase [EC: 1.5 .99 .8$]$ (N) " (0f5d3d4b481b65cb949b9c3998839ebd73b6a641) gtcgtggatgtactggcetcggcgttcgacagtgcgggtcagcgttgttcggcgctgcgcgtgct gtgcctgcaagatgagattgccgaccacacgttgaaaatgctgcgcggcgcaatggccgaatgcc ggatgggtaatccgggtcgcctgaccaccgatatcggtccagtgattgatagcgaagcgaaagcc aatattgagcgccatattcagaccatgcgtagcaaaggccgtccggtgttccaggcggtgcggga aaacagcgaagatgcccgtgaatggcaaagcggcacctttgtcgccccgacgctgatcgaactgg atgactttgccgaattgcaaaaagaggtctttggtccggtgctgcatgtggtgcgttacaaccgt aaccagctaccagagctgatcgagcagattaacgcttccggttatggtctgacgcttggcgtcca tacgcgcattgatgaaaccatcgcccaggtcactggctcggcccatgttggtaacctgtatgtta accgtaatatggtgggcgcagtggttggtgtgcagccgttcggcggcgaagggttgtccggtacc gggccgaaagcaggcggtccgctctatctctaccgtctgctg

$>$ 22.1 |SOURCES $=\{\mathrm{KEY}=0 \mathrm{f} 5 \mathrm{~d} 3 \mathrm{~d} 4 \mathrm{~b} \ldots \mathrm{fw}, 2534-3180\} \mid$ ERRORS $=\{\} \mid$ SOURCE_1="eco:b1014 putA, ECK1005, JW0999, poaA, putC; fused DNA-binding transcriptional regulator/proline dehydrogenase/pyrroline-5-carboxylate dehydrogenase (EC:1.5.99.8 1.5.1.12); K00294 1pyrroline-5-carboxylate dehydrogenase [EC:1.5.1.12] K00318 proline dehydrogenase

[EC: 1.5 .99 .8$]$ (N) " (0f5d3d4b481b65cb949b9c3998839ebd73b6a641)

tgatcgcgtgcgcggggtgatgtttaccggttcaaccgaagtcgctacgttactgcagcgcaata tcgccagccgcctggacgctcagggtcgccetattccgetcatcgctgaaaccggcggcatgaac gcgatgattgtcgattcttcagcactgaccgaacaggtcgtcgtggatgtactggcctcggcgtt cgacagtgcgggtcagcgttgttcggcgctgcgcgtgctgtgcctgcaagatgagattgccgacc acacgttgaaaatgctgcgcggcgcaatggccgaatgccggatgggtaatccgggtcgcctgacc accgatatcggtccagtgattgatagcgaagcgaaagccaatattgagcgccatattcagaccat gcgtagcaaaggccgtccggtgttccaggcggtgcgggaaaacagcgaagatgcccgtgaatggc aaagcggcacctttgtcgcccgacgctgatcgaactggatgactttgccgaattgcaaaaagag gtcttggtccggtgctgcatgtggtgcgttacaaccgtaaccagctaccagagctgatcgagca gattaacgcttccggttatggtctgacgcttggcgtccatacgcgcattgatgaaaccatc

$>$ 3 $3.1 \mid$ SOURCES $=\{\mathrm{KEY}=0 \mathrm{f} 5 \mathrm{~d} 3 \mathrm{~d} 4 \mathrm{~b} \ldots \mathrm{fw}, 163-820\} \mid$ ERRORS $=\{\} \mid$ SOURCE_1="eco:b1014 putA, ECK1005, JW0999, poaA, putC; fused DNA-binding transcriptional regulator/proline dehydrogenase/pyrroline-5-carboxylate dehydrogenase (EC:1.5.99.8 1.5.1.12); K00294 1pyrroline-5-carboxylate dehydrogenase [EC:1.5.1.12] K00318 proline dehydrogenase [EC: 1.5 .99 .8$]$ (N) " (0f5d3d4b481b65cb949b9c3998839ebd73b6a641) cgctgctttctggcgcggccaatgagagcgatgaagcaccgactccggcagaggaaccacaccag ccattcctcgactttgccgagcaaatattgccccagtcggtttcccgcgccgcgatcaccgcggc 
ctatcgccgcccggaaaccgaagcggtttctatgctgctggaacaagcccgcctgccgcagccag ttgctgaacaggcgcacaaactggcgtatcagctggccgataaactgcgtaatcaaaaaaatgcc agtggtcgcgcaggtatggtccaggggttattgcaggagttttcgctgtcatcgcaggaaggcgt ggcgctgatgtgtctggcggaagcgttgttgcgtattcccgacaaagccacccgcgacgcgttaa ttcgcgacaaaatcagcaacggtaactggcagtcacacattggtcgtagcccgtcactgtttgtt aatgccgccacctgggggetgctgtttactggcaaactggtttccacccataacgaagccagcct ctcccgctcgctgaaccgcattatcggtaaaagcggtgaaccgctgatccgcaaaggtgtggata tggcgatgcgcctgatgggtgagcagttcgtcactggcgaaaccatcgcggaagcgttagccaat geccgca

$>r 4.1 \mid$ SOURCES $=\{\mathrm{KEY}=0 \mathrm{f} 5 \mathrm{~d} 3 \mathrm{~d} 4 \mathrm{~b} \mathrm{~b} . \mathrm{fw}, 3344-3963 ; \mathrm{KEY}=0 \mathrm{f} 5 \mathrm{~d} 3 \mathrm{~d} 4 \mathrm{~b} \ldots \ldots, \mathrm{fw}, 0-$ 40\} $\mid$ ERRORS=\{\}|SOURCE_1="eco:b1014 putA, ECK1005, JW0999, poaA, putC; fused DNA-binding transcriptional regulator/proline dehydrogenase/pyrroline-5-carboxylate dehydrogenase (EC: 1.5 .99 .8 1.5.1.12); K00294 1-pyrroline-5-carboxylate dehydrogenase [EC:1.5.1.12] K00318 proline dehydrogenase [EC:1.5.99.8] (N) "

(0f5d3d4b481b65cb949b9c3998839ebd73b6a641)

aagtgcgctggcagtgacgctcgcgcgtcaggatgcaaagtatccggtcgatgcgcagttgaaag ccgcattgactcagccgctaaatgcactgcgggaatgggcagcaaatcgtccagaattgcaggcg ttatgtacgcaatatggcgagctggcgcaggcaggaacacaacgattgctgccggggccgacggg tgaacgcaacacctggacgctgctgccgcgtgagcgcgtgttgtgtattgccgatgatgagcagg atgcgctgactcagctcgccgccgtgctggcggtgggcagccaggtactgtggccggatgacgcg ctgcatcgtcagttagtgaaggcattgccatcggcagtcagcgaacgtattcaactggcgaaagc ggaaaatataaccgctcaaccgtttgatgcggtgatcttccacggtgattcggatcagcttcgcg cattgtgtgaagcagttgccgcgcgggatggcacaattgtttcggtgcagggttttgcccgtggc gaaagcaatatccttctggaacggctgtatatcgagcgttcgctgagtgtgaataccgctgccgc tggcggtaacgccagcttaatgactataggttaaatgggaaccaccaccatgggggttaagctgg acgacgcga

>r5.1 | SOURCES $=\{\mathrm{KEY}=0 \mathrm{f} 5 \mathrm{~d} 3 \mathrm{~d} 4 \mathrm{~b} \ldots \mathrm{fw}, 3400-3963 ; \mathrm{KEY}=0 \mathrm{f} 5 \mathrm{~d} 3 \mathrm{~d} 4 \mathrm{~b} \ldots \ldots, \mathrm{fw}, 0-$ 118 $\mid$ ERRORS=\{\}|SOURCE_1="eco:b1014 putA，ECK1005，JW0999，poaA，putC; fused DNA-binding transcriptional regulator/proline dehydrogenase/pyrroline-5-carboxylate dehydrogenase (EC:1.5.99.8 1.5.1.12); K00294 1-pyrroline-5-carboxylate dehydrogenase [EC:1.5.1.12] K00318 proline dehydrogenase [EC:1.5.99.8] (N) "

(0f5d3d4b481b65cb949b9c3998839ebd73b6a641)

agttgaaagccgcattgactcagccgctaaatgcactgcgggaatgggcagcaaatcgtccagaa ttgcaggcgttatgtacgcaatatggcgagctggcgcaggcaggaacacaacgattgctgccggg gccgacgggtgaacgcaacacctggacgctgctgccgcgtgagcgcgtgttgtgtattgccgatg atgagcaggatgcgctgactcagctcgccgccgtgctggcggtgggcagccaggtactgtggecg gatgacgcgctgcatcgtcagttagtgaaggcattgccatcggcagtcagcgaacgtattcaact ggcgaaagcggaaaatataaccgctcaaccgtttgatgcggtgatcttccacggtgattcggatc agcttcgcgcattgtgtgaagcagttgccgcgcgggatggcacaattgtttcggtgcagggtttt gcccgtggcgaaagcaatatccttctggaacggctgtatatcgagcgttcgctgagtgtgaatac $[\ldots]$ 


\section{APPENDIX F}

\section{METASIM 454 EXCERPT}

$>\mathrm{r} 1.1$ |SOURCES $=\{\mathrm{KEY}=0 \mathrm{f} 5 \mathrm{~d} 3 \mathrm{~d} 4 \mathrm{~b} . \ldots, \mathrm{fw}, 1824-$

$2078\} \mid$ ERRORS $=\left\{22 \_1: T, 38 \_1: T, 57 \_1: G, 72 \_1: G, 89 \_1: T, 119 \_1: T, 153 \_1: G, 206 \_1: A\right\} \mid S O U R C E \_1="$ eco : b

1014 putA, ECK1005, JW0999, poaA, putC; fused DNA-binding transcriptional

regulator/proline dehydrogenase/pyrroline-5-carboxylate dehydrogenase (EC:1.5.99.8

1.5.1.12); K00294 1-pyrroline-5-carboxylate dehydrogenase [EC:1.5.1.12] K00318 proline

dehydrogenase [EC:1.5.99.8] (N)" (0f5d3d4b481b65cb949b9c3998839ebd73b6a641)

cgcgatctttacggtcacgggcgTcgacaactcggcagggTctggatctcgctaacgaacGaccg

cctggcctcgcGtctcctctgccetgctcTaatagtgcactgcaaaaatggcaggccttgTccaa

tgctggaacaaccggtagcggcaggtgagaGtgtcgcccgttattaaccctgcggaaccgaaaga

tattgtgggctatgtgcgtAgaagccacgccgcgtgaagtagaacaggcgctggaaagtgcggtt

aa

$>\mathrm{r} 2.1|\mathrm{SOURCES}=\{\mathrm{KEY}=0 \mathrm{f} 5 \mathrm{~d} 3 \mathrm{~d} 4 \mathrm{~b} \ldots \mathrm{f} \mathrm{fw}, 2006-2253\}| \mathrm{ERRORS}=\left\{18 \_1: \mathrm{G}, 59:-, 77 \_1: \mathrm{G}, 151 \_1: \mathrm{G}, 154:-\right.$

\}|SOURCE_1="eco:b1014 putA, ECK1005, JW0999, poaA, putC; fused DNA-binding

transcriptional regulator/proline dehydrogenase/pyrroline-5-carboxylate dehydrogenase

(EC:1.5.99.8 1.5.1.12); K00294 1-pyrroline-5-carboxylate dehydrogenase [EC:1.5.1.12]

K00318 proline dehydrogenase [EC:1.5.99.8] (N) "

( 0 f5d3d4b481b65cb949b9c3998839ebd73b6a641)

gaaagatattgtgggctatggtgcgtgaagccacgccgcgtgaagtagaacaggcgctggaagtg

cggttaataacgcGgccaatctggtttgccacgcctccggctgaacgcgcagcgattttgcaccg

cgctgccgtgctgatggaaagccGagtgcagcaactgattggtattctggtgcgtgaggccggaa

aaaccttcagtaacgccattgccgaagtgcgcgaagcggtcgattttctccac

$>\mathrm{r} 3.1$ SOURCES $=\{\mathrm{KEY}=0 \mathrm{f} 5 \mathrm{~d} 3 \mathrm{~d} 4 \mathrm{~b} \ldots \ldots \mathrm{fw}, 3721-3963 ; \mathrm{KEY}=0 \mathrm{f} 5 \mathrm{~d} 3 \mathrm{~d} 4 \mathrm{~b} . \ldots, \mathrm{fw}, 0-$

$16\} \mid$ ERRORS $=\left\{18 \_1: C, 26 \_1: A, 95 \_1: C, 134 \_1: A, 139 \_1: C, 168 \_1: A, 190:-\right.$

,200_1:T,228_1:C,243_1:C\}|SOURCE_1="eco:b1014 putA，ECK1005，JW0999, poaA, putC; fused DNA-binding transcriptional regulator/proline dehydrogenase/pyrroline-5-carboxylate dehydrogenase (EC:1.5.99.8 1.5.1.12); K00294 1-pyrroline-5-carboxylate dehydrogenase [EC:1.5.1.12] K00318 proline dehydrogenase [EC:1.5.99.8] (N) "

(0f5d3d4b481b65cb949b9c3998839ebd73b6a641)

aactggcgaaagcggaaaaCtataaccgActcaaccgtttgatgcggtgatcttccacggtgatt cggatcagcttcgcgcattgtgtgaagcagttgCccgcgcgggatggcacaattgtttcggtgca gggttttgAcccgtCggcgaaagcaatatccttctggaacggctAgtatatcgagcgttcgctga ggtgaataccgTctgccgctggcggtaacgccagcttaatCgactataggttaaatCgggaacca ccacca

$>r 4.1 \mid$ SOURCES $=\{$ KEY $=0 f 5 d 3 d 4 b .$. f fw, $1209-1469\} \mid$ ERRORS $=\left\{20:-, 40 \_1: C, 44 \_1: C, 175:-, 198:-\right.$ ,209_1:A,237_1:T\}|SOURCE_1="eco:b1014 putA, ECK1005, JW0999, poaA, putC; fused DNAbinding transcriptional regulator/proline dehydrogenase/pyrroline-5-carboxylate dehydrogenase (EC:1.5.99.8 1.5.1.12); K00294 1-pyrroline-5-carboxylate dehydrogenase 
[EC:1.5.1.12] K00318 proline dehydrogenase [EC:1.5.99.8] (N) " (0f5d3d4b481b65cb949b9c3998839ebd73b6a641)

caggcttatcaaaaacgctgccgttggtgatcgattacctCgattCgatctcgccacccgcagcc gtcgccgtctgatgattcgcctggtgaaaggcgcgtactgggatagtgaaattaagcgtgcgcag atggacggccttgaaggttatccggtttatacccgcaaggtgtatacgacgtttcttatctcgcc tgtcgaaaaagctgActggcggtgccgaatctaatctacccgcTagttcgcgacgcacaacgccc

a

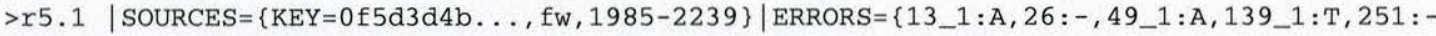
\}|SOURCE_1="eCo:b1014 putA, ECK1005, JW0999, poaA, putC; fused DNA-binding transcriptional regulator/proline dehydrogenase/pyrroline-5-carboxylate dehydrogenase (EC:1.5.99.8 1.5.1.12); K00294 1-pyrroline-5-carboxylate dehydrogenase [EC:1.5.1.12] K00318 proline dehydrogenase [EC:1.5.99.8] (N)"

(0f5d3d4b481b65cb949b9c3998839ebd73b6a641)

cgttattaaccctgAcggaaccgaaagtattgtgggctatgtgcgtgaagAccacgccgcgtgaa gtagaacaggcgctggaaagtgcggttaataacgcgccaatctggtttgccacgcctccggctga acgcgcagcgaTttttgcaccgcgctgccgtgctgatggaaagccagatgcagcaactgattggt attctggtgcgtgaggccggaaaaaccttcagtaacgccattgccgaagtgcgcgaaggg $>r 6.1$ SOURCES $=\{$ KEY $=0 f 5 d 3 d 4 b \ldots, b w, 1905-$ $2155\} \mid$ ERRORS $=\left\{2 \_1: \mathrm{A}, 24 \_1: \mathrm{T}, 44 \_1: \mathrm{T}, 89 \_1: \mathrm{A}, 95 \_1: \mathrm{T}, 203 \_1: \mathrm{C}, 205 \_1: \mathrm{T}\right\} \mid \mathrm{SOURCE} \_1=$ "eco $: \mathrm{b} 1014$ putA, ECK1005, JW0999, poaA, putC; fused DNA-binding transcriptional regulator/proline dehydrogenase/pyrroline-5-carboxylate dehydrogenase (EC:1.5.99.8 1.5.1.12); K00294 1pyrroline-5-carboxylate dehydrogenase [EC:1.5.1.12] K00318 proline dehydrogenase [EC: 1.5 .99 .8$] \quad(\mathrm{N}) "(0 \mathrm{f} 5 \mathrm{~d} 3 \mathrm{~d} 4 \mathrm{~b} 481 \mathrm{~b} 65 \mathrm{cb} 949 \mathrm{~b} 9 \mathrm{c} 3998839 \mathrm{ebd} 73 \mathrm{~b} 6 \mathrm{a} 641)$ TTTACCATCAGCACGGCAGCGCGGTGTCAAAATCGCTGCGCGTTCAGTCCGGAGGCGTGGCAAAC CAGATTGGCGCGTTATTAACCGCACTTTACCAGCGTCCTGTTCTACTTCACGCGGCGTGGCTTCA CGCACATAGCCCACAATATCTTTCGGTTCCGCAGGGTTAATAACGGGCGACATCTCACCTGCCGC TACCGGTTGTTCCACGCTATTGGCAAGGCCTGCCATTTTTGCAGTGCACTATTGAGCAGGGC $>r 7.1$ SOURCES $=\{$ KEY $=0 f 5 d 3 d 4 b \ldots, b w, 566-$

822 $\}$ ERRORS $=\left\{22 \_1: A, 52 \_1: A, 86 \_1: C, 90 \_1: T, 176 \_1: A, 216 \_1: G, 230 \_1: C\right\} \mid$ SOURCE_1 = "eco: b1014 putA, ECK1005, JW0999, poaA, putC; fused DNA-binding transcriptional regulator/proline dehydrogenase/pyrroline-5-carboxylate dehydrogenase (EC:1.5.99.8 1.5.1.12); K00294 1pyrroline-5-carboxylate dehydrogenase [EC:1.5.1.12] K00318 proline dehydrogenase [EC: 1.5 .99 .8$]$ (N) " (0f5d3d4b481b65cb949b9c3998839ebd73b6a641) CTTGCGGGCATTGGCTAACGCTTACCGCGATGGTTTCGCCAGTGACGAACTGCTACACCCATCAG GCGCATCGCCATATCCACACCTTTCGCGGTATCAGCGGTTCACCGCTTTTACCGATAATGCGGTT CAGCGAGCGGGAGAGGCTGGCTTCGTTATGGGTGGAAACCAGTTTGCCAGTAAAACAGCAGCCCC CAGGTGGCGGCATTAACAAACAGTGACGGGGCTACGACCAATCGTGTGACTGCCAGTTACCGTTG CTG

$[\ldots]$ 


\section{REFERENCES}

Altschul, Stephen F., et al. "Gapped BLAST and PSI-BLAST: A New Generation of Protein Database Search Programs." Nucleic Acid Res. 25 (1997): 3389-3402.

Applied Biosystems. Applied Biosystems Product Catalog. 22 March 2010. Web. 22 March 2010 <https://products.appliedbiosystems.com/ab/en/US/adirect/ab>.

Blattner, F. R., et al. "The Complete Genome Sequence of Escherichia coli K12." Science (1997): 1453-1462.

Chaisson, Mark, Pavel Pevzner and Haixu Tang. "Fragment Assembly with Short Reads." Bioinfomatics 20.13 (2004): 2067-2074.

Cormen, Thomas H., et al. Introduction to Algorithms. 2nd ed. New York: McGraw Hill, 2000.

Darnell, James, Harvey Lodish and David Baltimore. Molecular Cell Biology. 2nd ed. New York: Scientific American Books, 1990.

de Bruijn, N. G. "A Combinatorial Problem." Koninklijke Nederlandse Akademie v. Wetenschappen 49 (1946): 758-764.

DiGuistini, Scott, et al. "De Novo Genome Sequence Assembly of a Filamentous Fungus Using Sanger, 454 and Illumina Sequence Data." Genome Biology 10 (2009): R94.

Ewing, Brent and Phil Green. "Base-Calling of Automated Sequencer Traces UsingPhred. II. Error Probabilities." Genome Res. (1998): 186-194. 
Ewing, Brent, et al. "Base-Calling of Automated Sequencer Traces Using Phred.

I. Accuracy Assessment." Genome Res. 8 (1998): 175-185.

Gilbert, W. and A. Maxam. "The Nucleotide Sequence of the Lac Operator." Proc Natl Acad Sci U.S.A. 12.70 (1973): 3581-3584.

Gross, J. L. and J. Yellen. Handbook of Graph Theory. Boca Raton: CRC Press LLC, 2004.

Idury, Ramana M. and Michael S. Waterman. "A New Algorithm for DNA Sequence Assembly." Journal of Computational Biology 2.2 (1995): 291306.

Kyoto University Bioinformatics Center. GenomeNet. 22 March 2010. Web. 22 March $2010<$ http://www.genome.jp/>.

Lehninger, Albert L., David L. Nelson and Michael M. Cox. Principles of Biochemistry. 2nd ed. New York: Worth Publishers, 1993.

leipzig. Standardized-Velvet-Assembly-Report - Project Hosting on Google Code.

22 March 2010. Web. 22 March 2010

<http://code.google.com/p/standardized-velvet-assembly-report/>.

Marguiles, Marcel, et al. "Genome Sequencing in Open Microfabricated High Density Picoliter Reactors." Nature 437 (2005): 376-380.

Maxam, A. M. and W. Gilbert. "A New Method for Sequencing DNA." Proc Natl Acad Sci U S A. 2.74 (1977): 560-564.

NCBI. FASTA Format Description. 22 March 2010. Web. 22 March 2010 <http://www.ncbi.nlm.nih.gov/blast/fasta.shtml>. 
—. National Center for Biotechnology Information. 22 March 2010. Web. 22

March $2010<$ http://www.ncbi.nlm.nih.gov/>.

Pevzner, P. A. "1-Tuple DNA Sequencing: Computer Analysis." J Biomol Struct Dyn 7 (1989): 63-73.

Pevzner, Pavel A., Haixu Tang and Michael S. Waterman. "An Eulerian Path Approach to DNA Fragment Assembly." PNAS 98.17 (2001): 9748-9753.

Richter, Daniel C., et al. "MetaSim—A Sequencing Simulator for Genomics and Metagenomics." PLoS ONE 3.10 (2008): e3373.

Roche Diagnostics Co. Products \& Solutions - Syetem Benefits : 454 Life Sciences, a Roche Company. 22 March 2010. Web. 22 March 2010 <http://454.com/products-solutions/system-benefits.asp>.

Ronaghi, M., M. Uhlén and P. Nyrén. "A Sequencing Method Based on RealTime Pyrophosphate." Science (1998): 363, 365.

Sanger, Fredrick, S. Nicklen and A. R. Coulson. "DNA Sequencing with ChainTerminating Inhibitors." Proc Natl Acad Sci U.S.A. 12.74 (1977): 54635467.

Schatz, Michael C., et al. "Hawkeye: An Interactive Visual Analytics Tool for Genome Assemblies." Genome Biology 8 (2007): R34.

Tamarin, Robert H. Principles of Genetics. 4th ed. Dubuque: Wm. C. Brown Publishers, 1993.

Watson, James D. and Francis H. Crick. "Genetical Implications of the structure of Deoxyribonucleic Acid." Nature 171 (1953): 964-967. 
Watson, James D. and Franics H. Crick. "A Structure for Deoxyribose Nucleic Acid." Nature 171 (1953): 737-738.

Zerbino, Daniel R. and Ewan Birney. "Velvet: Algorithms for De Novo Short Read Assembly Using de Bruijn Graphs." Genome Research 18 (2008): 821829. 